Check for updates

Cite this: Chem. Commun., 2020, 56,11735

Received 22nd May 2020,

Accepted 19th August 2020

DOI: $10.1039 / \mathrm{d} 0 \mathrm{cc} 03659 \mathrm{e}$

rsc.li/chemcomm

\section{Evolution of metal organic frameworks as electrocatalysts for water oxidation}

\author{
Subhabrata Mukhopadhyay, (D) Olivia Basu, (D) Rajendar Nasani (D) and \\ Samar K. Das (D)*
}

\begin{abstract}
In the last two decades, metal organic frameworks (MOFs) have been extensively investigated to develop heterogeneous electrocatalysts for water oxidation (WO). The scope of reticular synthesis, enormous surface area and accessible internal volume of MOFs make them promising candidates for catalysis. However, low electrical conductivity, slow mass transport and lack of stability restrict the scope of MOFbased WO. In recent times, various material designing approaches, e.g., the introduction of mixed metal and multi-metal systems, ligand engineering, guest@MOF composite formation, preparation of thin films, MOF composite formation with conducting carbon-based materials, metal oxides, polymers and layered compounds, etc. have emerged as an effective means to counteract the aforementioned limitations. This feature article critically discusses the common MOF-based material designing strategies with respect to electrochemical WO and provides a platform to understand the potential of MOFs to prepare a sophisticated hybrid electrocatalyst for WO.
\end{abstract}

\title{
Introduction
}

For the past few decades, fossil fuels have been our main energy source, and are not only depleting at an alarming rate but have also been of major environmental concern. Besides, looking at rising socioeconomic standards and environmental concerns along with technological advancements, it is evident that with

School of Chemistry, University of Hyderabad, Hyderabad-500046, India. E-mail: skdas@uohyd.ac.in,samar439@gmail.com

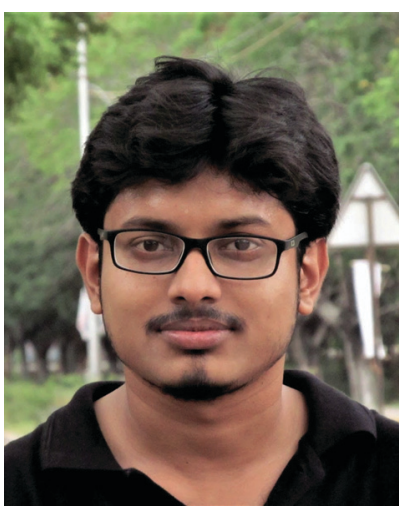

Subhabrata Mukhopadhyay
Subhabrata Mukhopadhyay, received his MSc degree in Chemistry from the Indian Institute of Technology, Roorkee (India). Later, he joined Prof. Samar K. Das' research group as a doctoral student, in the year of 2014. His doctoral work comprised synthesis and electrochemical studies of metalorganic framework (MOF) derived materials for application in alternate energy sources. At present, he is working as a postdoctoral fellow with Dr Idan Hod at the Ben-Gurion University of the Negev, Israel. He is currently working on electrochemical $\mathrm{CO}_{2}$ reduction by MOF-based materials. growing energy consumption, the urge for the generation and efficient storage of clean and renewable energy is also growing exponentially. ${ }^{1-3}$

Of the many available means, harnessing solar energy is the most promising option, since it has the capacity to sustain the entire planet. A two-step process, which involves (a) the conversion of solar energy to electrical energy using a photovoltaic cell and (b) storage of the electrical energy in the form of chemical energy of molecules, cumulatively constructs a path to generate fuel using sunlight. ${ }^{3-7}$ It is the second step of this

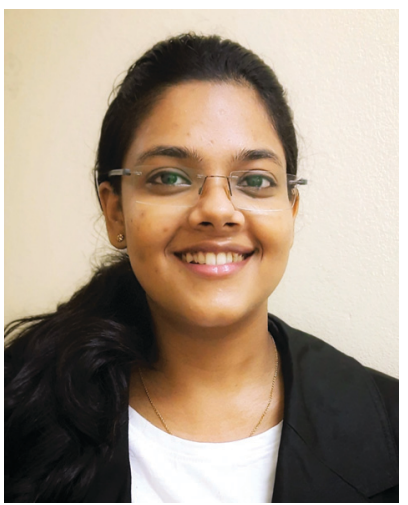

Olivia Basu
Olivia Basu obtained her BSc degree in Chemistry from the Presidency University, Kolkata (India) in 2015. She completed her MSc from the University of Hyderabad (India) in 2017, where she worked on hybrid composites of metalloporphyrins and metal-organic frameworks (MOFs) for application in OER catalysis. She is currently a doctoral student with the research group of Prof. Samar K. Das and her research interest lies in designing functional materials from metal organic frameworks (MOFs) and other supramolecular systems. 


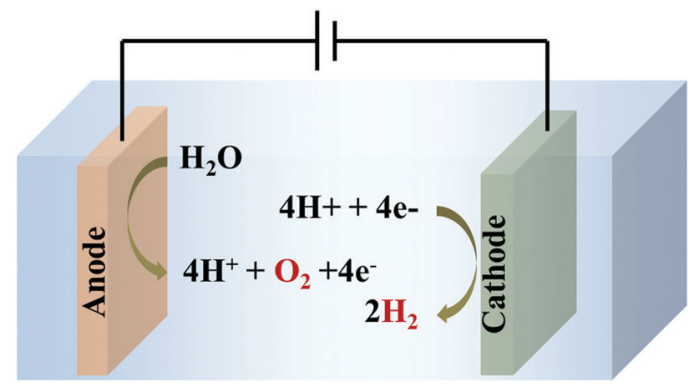

Scheme 1 Electrochemical water splitting to generate $\mathrm{O}_{2}$ and $\mathrm{H}_{2}$ by simultaneous water oxidation (at the anode) and water reduction (at the cathode), respectively

energy conversion process where electrochemical water splitting (WS) can play the prime role; here, the electrical energy is stored in the chemical bonds of $\mathrm{H}_{2} \cdot \mathrm{H}_{2}$ being a clean source of high-density energy, has been considered as a potential replacement of fossil fuels. Hence, drawing inspiration from photosynthesis - the most efficient solar energy harvesting process, scientists have been relentlessly optimizing the electrochemical OER catalysts over the past few decades. ${ }^{6,8-28}$

Electrochemical WS comprises two fundamental processes water oxidation (WO)/oxygen evolution reaction (OER) (an anodic reaction), and hydrogen evolution reaction (HE R) (a cathodic reaction), as shown in Scheme 1 .

The OER (eqn (1)) is thermodynamically more demanding than the HER (eqn (2)). ${ }^{7,22,29-35}$ The OER involves ' $\mathrm{O}-\mathrm{H}$ ' bond breaking and ' $\mathrm{O}-\mathrm{O}$ ' bond making through a series of multistep processes, which again involves several intermediates and an overall transfer of $4 \mathrm{e}^{-} / 4 \mathrm{H}^{+}$. It needs to overcome a high kinetic energy barrier and has a high overpotential requirement. ${ }^{13,27,36-46}$ Thus, it is crucial to develop a robust and efficient OER catalyst to accomplish overall water splitting in a satisfactory manner. Of note, WO is also important for several crucial processes related to the alternate energy research. For various electrochemical processes, WO acts as the source of protons, which is essential to advance the overall process without the participation of a sacrificial agent. Thus, it is of prime importance to develop efficacious and stable OER catalysts, to make the complete system of solar energy harvesting viable.

$$
\begin{aligned}
& 2 \mathrm{H}_{2} \mathrm{O} \rightarrow \mathrm{O}_{2}+4 \mathrm{H}^{+}+4 \mathrm{e}^{-} \\
& 4 \mathrm{H}^{+}+4 \mathrm{e}^{-} \rightarrow 2 \mathrm{H}_{2}
\end{aligned}
$$

$$
2 \mathrm{H}_{2} \mathrm{O} \rightarrow \mathrm{O}_{2}+2 \mathrm{H}_{2} \quad E_{\text {reaction }}=-1.23 \mathrm{~V}
$$

\section{Parameters relevant to OER catalysis}

The efficiency of an electrochemical catalyst in general is defined in terms of (1) overpotential $(\eta)$ requirement, (2) obtained current density, (3) faradaic efficiency (FE), (4) turnover number (TON), (5) turnover frequency (TOF) and (6) durability under operational conditions. As has already been mentioned, the multistep OER process has a high overpotential requirement. Therefore, it is always preferred for a catalyst to perform the OER with minimum overpotential. In addition, for real life application, high and stable catalytic current density is crucial. Thus, the overpotential $(\eta)$ requirement to obtain a current density $(j)$ of $10 \mathrm{~mA} \mathrm{~cm}{ }^{-2}$, i.e., $\eta_{10}$ is considered as an important parameter to define the efficiency of a catalyst. ${ }^{6,7,24}$ $\mathrm{FE}$ is a measure of the conversion of electrical energy into chemical energy during the OER. TON and TOF are two key parameters, of which TON gives an estimate of the amount of product produced (in this case oxygen) per active catalytic site in the complete lifetime of the catalyst and TOF gives the amount of oxygen produced per active catalytic site per unit time. Defining TOF is a more acceptable means of quantifying the efficiency of an OER catalyst while TON relates to the performance of a catalyst with its stability under operational

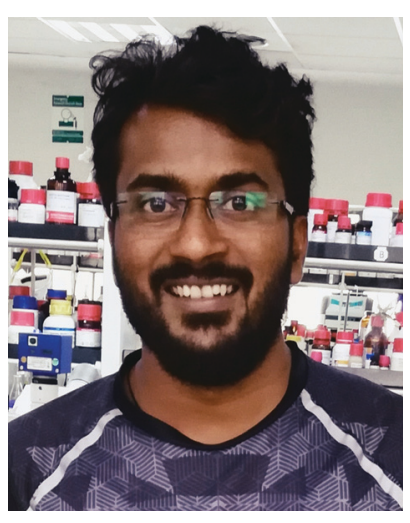

Rajendar Nasani
Rajendar Nasani received his MSc degree in 2008 from the Osmania University. He pursued his PhD with Prof. Suman Mukhopadhyay (2015), from IIT Indore. His doctoral work was on metal-nitrile/tetrazolate coordination complexes and frameworks. In 2016 he received the National Postdoctoral Fellowship by DST-SERB, and joined Prof. Sanjit Konar's research group at IISER Bhopal India. There, he worked on rare earth metalorganic frameworks and also on radical based magnetic materials. Presently, he is working with Prof. Samar K. Das as a Dr D. S. Kothari postdoctoral fellow. His current research interests include exploration of viologen-POM composites for energy storage applications.

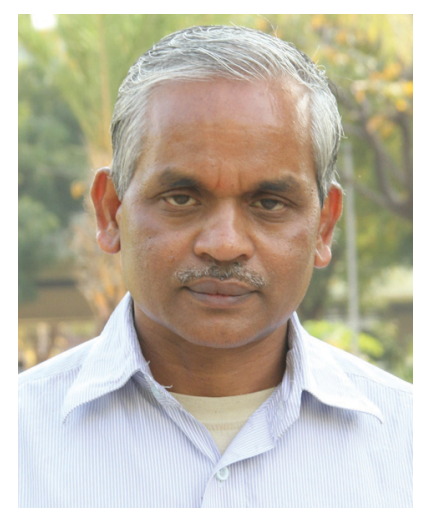

Samar K. Das
Samar K. Das obtained his PhD from IIT Kanpur (1995). His first postdoctoral work was on zeolite included compounds with Professor Prabir K. Dutta at the Ohio State University, USA. Later, he worked on giant polyoxometalate clusters with Professor Achim Mueller at Universitaet Bielefeld, Germany. He is currently a Professor at the School of Chemistry, University of Hyderabad (India). He has worked in areas like dithiolenebased materials, polyoxometalates (POMs), MOFs, etc. with the primary objective of developing functional materials of potential applications. Recently, his research group has focused on developing POM- and MOF-based materials for OER and HER catalysis. 
conditions. Stability is another important factor for real-life applicability of a catalyst. ${ }^{4-51}$ The stability of an OER electrocatalyst can be determined by a combination of controlled experiments and various characterization techniques (electrochemical and spectroscopic). Additionally, it is worth mentioning that determination of the Tafel slope is an important tool to elucidate the mechanism involved. ${ }^{13,16}$

\section{Electrocatalysts for the OER}

OER catalysts can be classified as homogenous catalysts (molecular) and heterogeneous catalysts (with extended structure), based on the physical state of the active species. However, during the course of time, this classification appeared to be less appropriate as many homogeneous catalysts were reported not to behave as a molecular catalyst, whereas various heterogeneous catalysts were reported in which the catalytic OER was performed by a molecular functional unit present within the structure. ${ }^{3,5,36}$ Hence, it is more important to understand if a proposed catalyst is a true catalyst in nature, which can perform WO by itself, or if it gets converted to any secondary extended structure under the operational conditions of WO. If a catalyst can perform WO as a true catalyst, whether in a homogeneous or heterogeneous manner, it would be of more interest than the ones which get converted to a secondary extended structure (mostly oxides) under highly oxidative environment of electrochemical OER. ${ }^{6,7,52,53}$

In this regard, MOFs and MOF-derived materials are being considered to possess enormous potential, since MOFs behave as a heterogeneous assembly of numerous molecular functional units packed in a relatively small volume, and all of these functional units can ideally participate in OER electrocatalysis, similar to independent molecular entities. ${ }^{54-56}$ The two major structural advantages of MOFs are their extraordinarily high surface area, and permanent porosity. This allows the reactants (in this case, water molecules) and products (in this case, oxygen) to easily diffuse to and from the catalytic sites in the MOF. Additionally, by careful choice of the metal ion and organic linker, it is possible to fine-tune the size and nature of the pores and channels existing inside MOFs. Now, since most of the MOFs have a highly crystalline structure with self-repeating units, it provides a scope for understanding the structure-function relationship of MOF-based materials. However, it should also be mentioned that many of the pristine MOFs suffer from the shortcoming of significantly low electrical conductivity and in a few cases, lack of hydrophilicity inside the pores. Therefore, designing an efficient OER catalyst out of MOFs would require a careful consideration of a few points - (1) the transport of both charge and mass through the framework should be well balanced; (2) the transport of reactants/products and charge should take place faster than the rate of catalysis - so that the catalyst can perform to its optimum level and its efficiency is not limited by mass transport or charge conduction. Over the past few years, several studies on the mechanistic details of the charge transfer phenomenon in MOFs have been carried out, which can help as a guide to design better electrocatalysts. ${ }^{45,57-59}$

Various catalysts have been developed for the OER in the last few decades. ${ }^{26,37,48,60-64}$ In a parallel growing field, enormous progress has been made in the area of MOFs and MOF derived materials. ${ }^{19,23,36,47,62-67}$ But, the connection between the field of designing a WO catalyst and a MOF was occasional in the initial stages. ${ }^{24,47,56,68,69}$ In recent years, a few review articles in the field of heterogeneous water splitting, ${ }^{7,70}$ electrochemical applications of MOFs, ${ }^{51,64,71-74}$ electrochemical OER of MOFs, ${ }^{25,75}$ etc. have portrayed the scope of the MOF-based OER. However, the evolution of MOF-based material designing strategies to prepare an OER catalyst, within the period of the last five years is highly interesting and requires periodical reviewing. This feature article mainly focuses on this constant development of material designing strategies to prepare MOFbased electrocatalysts for the OER. For the sake of clarity, the discussion will be divided under four different major headings: (1) pristine MOFs and its structural engineering for OER catalysis; (2) guest@MOF composites for electrochemical OER; (3) MOF derived hybrid materials (such as, MOF supported systems, thin films, etc.) and (4) hybrid materials derived by pyrolysis of MOFs. The several types of OER electrocatalysts prepared using a MOF are schematically represented in Scheme 2.

\section{Pristine MOFs and its structural engineering for OER catalysis}

MOFs, which are a combination of metal nodes and connecting organic linkers (any bi- or polydentate organic ligand), leave us with a plethora of opportunities to explore. Furthermore, being highly porous and crystalline materials, they offer well-arranged and easily accessible catalytic sites, which makes it comparatively easy to assess the reaction mechanism. With growing

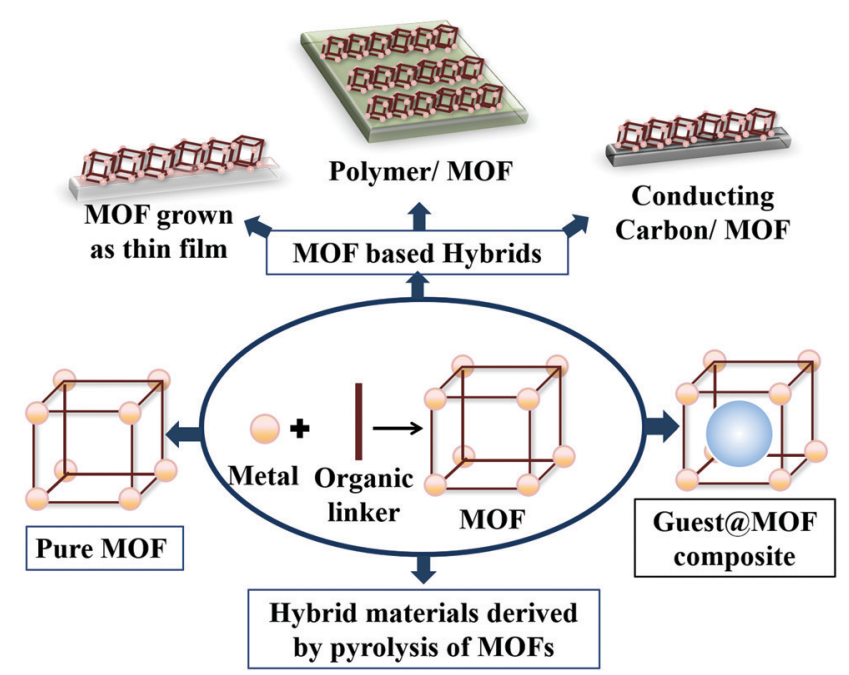

Scheme 2 Design strategy of MOF-based water oxidation (WO) catalysts by using pure MOF, guest@MOF, MOF-based hybrid materials and materials derived by pyrolysis of MOFs. 
interest in the field of designing and fabricating MOF-based electrocatalysts, Wang et al. prepared a cobalt containing zeolitic imidazolate framework (CoZIF-9) $\left\{\left[\mathrm{Co}(\text { bim })_{2}\right]\right\}$ with a benzimidazolate (bim) linker. ${ }^{46}$ A careful structural analysis revealed that the fabricated electrocatalyst comprised of imidazolate bridged $\mathrm{Co}^{\mathrm{II}}$ ions tetrahedrally connected to each other. Interestingly, the choice of benzimidazole over imidazole during the fabrication of the MOF was found to be not only crucial in increasing the stability of the MOF under high electrochemical OER anodic potential but also having an influence on the catalytic property of the MOF as was supported by DFT calculations and molecular simulations. On the other hand, in the majority of cases, the coordination environment around the catalytic centre was found to affect its electrocatalytic activity. One such study was conducted by Lu et al. on Co-benzotriazole MOF systems: MAF-X27-Cl and MAF-X27-OH. ${ }^{76}$ It can be seen from the respective molecular formulas $-\left[\mathrm{Co}_{2}(\mu-\mathrm{Cl})_{2}(\mathrm{btta})\right]$ for MAF-X27-Cl and $\left[\mathrm{Co}_{2}(\mu-\mathrm{OH})_{2}(\mathrm{btta})\right]$ for MAF-X27-OH $\left(\mathrm{H}_{2}\right.$ bbta $=$ $1 H, 5 H$-benzo(1,2- $\left.d: 4,5-d^{\prime}\right)$ bistriazole), that the only difference between these two sister MOFs lies in the coordination sphere of the active cobalt catalytic centre. From the experimental outcome, it was shown that the presence of $\mu-\mathrm{OH}$ ligand made MAF-X27-OH a superior OER catalyst than MAF-X27-Cl.

It is well known that different metal ions with their specific electronic structures and redox properties may exhibit electrocatalytic activity with different magnitude. In other words, for a given linker combination in a MOF, a change in the metal node may lead to a change in the catalytic activity as explained by Gong et al. ${ }^{77}$ in one of their reports. They fabricated three MOFs from three different metal nodes, i.e., $\mathrm{Co}, \mathrm{Cu}$ and $\mathrm{Zn}$, and a common polydentate organic linker L ([4-(5-(pyridin-4-yl)-4H1,2,4-triazol-3-yl) benzoic acid]) and commonly formulated as $\mathrm{M}_{2} \mathrm{~L}_{4} \cdot 3 \mathrm{H}_{2} \mathrm{O}$, with a common topology $4^{4}$-sql. Later, on careful electrocatalytic analysis, it was revealed that a MOF with a cobalt node showed better activity compared to that of the copper MOF; the zinc containing MOF stood inactive. Though a detailed mechanism was not provided, the results were attributed to difference in coordinative unsaturation around the metal centre which is mostly being controlled by the electronic structure of the metal centre. This clearly points out that a change in the node leads to a change in activity. Inspired from such a metal node dependent activity study, interest in designing and fabricating MOFs with complex node systems, like polynuclear (homo- and/or hetero-nuclear) nodes and mixed metal nodes, has increased. A few of those studies have been presented in later sections.

\section{1a. MOFs with polynuclear (metal cluster) nodes}

Inspired by different inorganic OER catalysts having multimetallic nodes, Gutiérrez-Tarriño et al. prepared a MOF formulated as $\left\{\left[\mathrm{Co}_{2}(\mathrm{BTC}) 1,3(\mathrm{DMF})_{2}\right]-\left[\mathrm{Co}_{2}(\mathrm{BTC}) 1,3(\mathrm{DMF})_{2} \mathrm{py}\right]\right\}$ $\left(\mathrm{Co}_{2}-\mathrm{MOF}\right)$ which constituted of two dissimilar binuclear secondary building units (SBUs); out of which, one of the SBUs was made up of two crystallographically independent cobalt centers. $^{78}$ Unfortunately, chemical stability studies revealed that the $\mathrm{Co}_{2}$-MOF was unstable in the presence of protic solvents like ethanol, water and their mixture, and was readily being hydrolysed to a different mononuclear cobalt-MOF. But it could be evaded by dispersing $\mathrm{Co}_{2}$-MOF in an alcoholic solution of Nafion. Interestingly, Nafion in the system not only enhanced protic solvent stability of the $\mathrm{Co}_{2}$-MOF catalyst but also assisted in improving its adherence to graphitic electrodes. Besides, electrocatalytic studies divulged that, $\mathrm{Co}_{2}-\mathrm{MOF}$ displayed superior water oxidation TOF values compared to that of a similar mononuclear Co-MOF, showing the advantage of fabricating polynuclear MOFs. Similarly, there is a recent report from Ibrahim et al., presenting a Co-trinuclear metal node containing MOF $\left\{\left[\mathrm{Co}_{3}(\mathrm{pyz})(\mathrm{fa})_{3}(\mathrm{dmso})_{2}\right] 2 \mathrm{H}_{2} \mathrm{O}\right\}_{n}$, (pyz = pyrazine, fa $=$ fumarate, dmso = dimethyl sulfoxide), that demonstrates notable performance towards the electrochemical OER in neutral $\mathrm{pH}^{79}$ Inspired from the multi-metal functional unit of plant photosystem, PS-II, Jiang et al. prepared a tetranuclear cobalt cluster $\left(\mathrm{Co}_{4} \mathrm{O}_{4}\right.$ cubane in the core) containing Co-citrate open framework (UTSA-16). ${ }^{80}$ This was the first report of a MOF-based OER catalyst, designed with a tetracobalt cubane type core. Owing to the synergistic cooperation of the $\left\{\mathrm{Co}_{4} \mathrm{O}_{4}\right\}$ cubane core with in situ formed high-valent cobalt centers and an open framework structure, UTSA-16 showed excellent OER activity, comparable with that of $\mathrm{RuO}_{2}$ and even surpassed its standard $\mathrm{Co}_{3} \mathrm{O}_{4}$ counterpart.

Apart from cobalt, researchers have also explored other transition metals in their quest to develop MOFs with polynuclear metal nodes. Recently, Wang et al. have developed a new alkaline stable MOF $\left[\mathrm{Ni}_{4}(\mathrm{OH})_{2}(\mathrm{NDC})_{3}\left(\mathrm{H}_{2} \mathrm{O}\right)_{2}\right] \cdot 2 \mathrm{H}_{2} \mathrm{O}$ (Ni-MOF), with a tetranuclear $\mathrm{Ni}_{4}\left(\mu^{3}-\mathrm{OH}\right)_{2}$ cluster as a metal node. ${ }^{81}$ This Ni-MOF performed quite well as an electrochemical catalyst for the OER. But, from several post-OER characterizations, it was found that this Ni-MOF underwent a phase transformation to form $\beta-\mathrm{Ni}(\mathrm{OH})_{2}$, such that both Ni-MOF and $\beta-\mathrm{Ni}(\mathrm{OH})_{2}$ coexisted in the same material. This new composite, formed by the partial leaching of the ligand, proved to be a better electrocatalyst for the OER as compared to $\beta-\mathrm{Ni}(\mathrm{OH})_{2}$ and Ni-MOF separately (Scheme 3). According to the authors, such a catalytic outcome might be a result of some synergistic

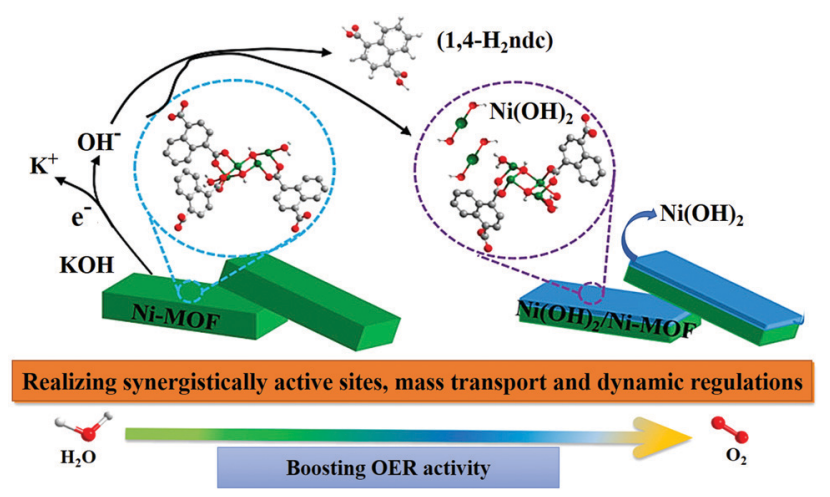

Scheme 3 A schematic representation of the transformation of Ni-MOF to $\mathrm{Ni}(\mathrm{OH})_{2} / \mathrm{Ni}-\mathrm{MOF}$ heterostructure and the synergistic catalytic OER process by $\mathrm{Ni}(\mathrm{OH})_{2} / \mathrm{Ni}-\mathrm{MOF}$. Adapted with permission from (ref. 81). Copyright (2020) American Chemical Society. 
a

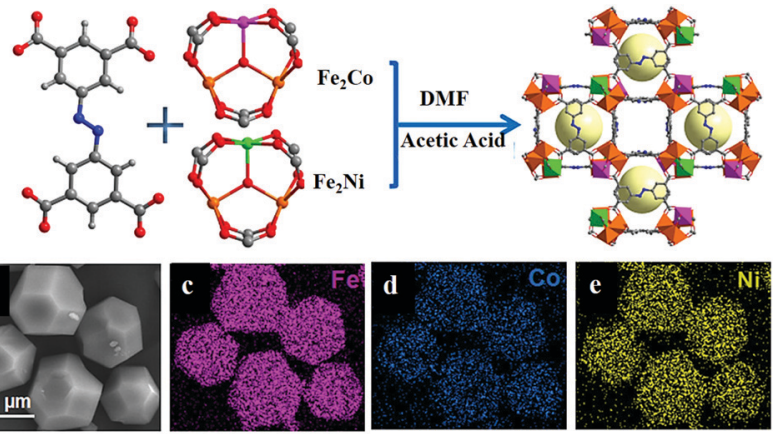

Fig. 1 (a) Schematic diagram of the synthesis of $\mathrm{PCN}-\mathrm{Fe}_{2} \mathrm{Co}-\mathrm{Fe}_{2} \mathrm{Ni}$. (b) Scanning electron microscopy (SEM) image and (c-e) corresponding elemental ( $\mathrm{Fe}$, $\mathrm{Co}$ and $\mathrm{Ni}$ ) mapping images for $\mathrm{PCN}-\mathrm{Fe}_{2} \mathrm{CO}-\mathrm{Fe}_{2} \mathrm{Ni}$. Adapted with permission from (ref. 82). Copyright (2019) American Chemical Society.

interaction between the two catalytically active species $\beta-\mathrm{Ni}(\mathrm{OH})_{2}$ and $\mathrm{Ni}_{4}\left(\mu_{3}-\mathrm{OH}\right)_{2}$. Additionally, the leaching of the ligand from the MOF created several void spaces, thereby increasing the specific surface area of the material. This facilitated the electrolyte and ion movement and exposed more active sites on the surface. In addition to this, the formation of $\beta-\mathrm{Ni}(\mathrm{OH})_{2}$ also improved the electrical conductivity of the material. Moving a step forward, in a different approach, Dong et al. employed a mixed-metal cluster strategy for synthesizing MOFs. ${ }^{82}$ In the first step, two heteronuclear MOFs were prepared PCN-250- $\mathrm{Fe}_{2} \mathrm{M}$ (M: $\mathrm{Co}$ or Ni) [heteronuclear cluster $\left.\mathrm{Fe}_{2} \mathrm{M}=\mathrm{Fe}_{2} \mathrm{M}\left(\mu_{3}-\mathrm{O}\right)\left(\mathrm{CH}_{3} \mathrm{COO}\right)_{6}\left(\mathrm{H}_{2} \mathrm{O}\right)_{3}\right]$. And in the second step, a new MOF $\mathrm{PCN}-\mathrm{Fe}_{2} \mathrm{Co}-\mathrm{Fe}_{2} \mathrm{Ni}$ was prepared by mixing both the heteronuclear clusters $\mathrm{Fe}_{2} \mathrm{Co}$ and $\mathrm{Fe}_{2} \mathrm{~N}$ (Fig. 1); a comparative study of electrocatalytic activity of these three materials was then conducted.

\section{1b. MOFs with mixed-nodes}

It has been found in several studies that the presence of different kinds of metals in a MOF tends to improve the electrocatalytic activity of the MOF towards the OER. A synergistic coupling between the metal centers has been suggested to be a driving force for such behaviour.

From a library of strategies, employing a metalloligand (e.g., metalloporphyrin) can be a very useful technique to incorporate active catalytic sites and thus to generate multi-metallic MOFs. In the year 2016, Usov et al. reported a Ni-porphyrin based MOF (PCN-224 (Ni)), where the metal nodes were made of a $\mathrm{Zr}$-oxo cluster while Ni-tetracarboxyphenylporphyrin (Ni-TCPP) acted as the metalloligand connecting the nodes. ${ }^{83}$ Even though it was proved that the Ni-porphyrin functioned as the active catalytic center, the $\mathrm{Zr}$-oxo metal nodes played an important role in the overall catalytic activity of PCN-224 (Ni), by increasing the hydrophilicity of the MOF. Another advantage of such systems is that the chances of degradation of the MOF by oxidative cleavage of the bond between the metal nodes and organic linker (in this case, metalloligand) is very low, since the metal ion of the metalloligand itself (and not the metal node) is supposed to be the catalytic site. Apart from in situ synthesis, post-synthetic modification (PSM) can also be a promising strategy for incorporating functionality into a MOF matrix. In a work by Maity et al., doping of $\mathrm{Co}^{\mathrm{II}}$ ions into a Cd-MOF crystal was performed simply by dipping the MOF in a $\mathrm{Co}\left(\mathrm{NO}_{3}\right)_{2}$ solution (in DMF). ${ }^{84}$ The final material thus formed by virtue of chemical bond formation between the $\mathrm{Co}^{\mathrm{II}}$ ions and the previously uncoordinated pyridine ligands of the Cd-MOF, was rendered active towards OER electrocatalysis. In order to study the effect of incorporating mixed-nodes in the same MOF structure, towards OER electrocatalysis, Gao et al. selected the Hofmann family of MOFs.$^{85}$ Hofmann MOFs can be designated by the formula $[\mathrm{M}] \mathrm{L}\left[\mathrm{M}^{\prime}(\mathrm{CN})_{4}\right]\left(\mathrm{M}\right.$ for $\mathrm{Fe}^{2+}, \mathrm{Co}^{2+}, \mathrm{Ni}^{2+}, \mathrm{Zn}^{2+} ; \mathrm{L}$ for pyridyl or dipyridyl derivatives; $\mathrm{M}^{\prime}$ for $\mathrm{Ni}^{2+}, \mathrm{Pd}^{2+}$, and $\mathrm{Pt}^{2+}$ ). As is evident from the formula, this MOF provides a wide scope of chemical tunability with all the possible combinations of metal ions and ligands. The authors utilized this feature to develop a series of Hofmann MOFs and studied their catalytic activity towards the OER. In the same year, another group of researchers reported a series of mixed-node MOFs with the formula $\mathrm{Co}_{x} \mathrm{Fe}_{1-x^{-}}$ MOF-74 $(0<x \leq 1)$. They were also successful in unveiling some of the mechanistic details of OER catalytic activity in such mixed-node MOFs ${ }^{86}$ According to their findings, the electron density on the cobalt nodes was larger in $\mathrm{Co}_{0.6} \mathrm{Fe}_{0.4}$-MOF-74 (MOF in the $\mathrm{Co}_{x} \mathrm{Fe}_{1-x}$-MOF-74 series having a better activity than Co-MOF-74) than its mono-metallic analogue, Co-MOF-74. Such a phenomenon was suggested to be a result of Fe-doping in the MOF, causing a shift in electron density from Fe to Co. This also explains the improved performance of the catalyst, since a higher electron density over the active catalytic center would facilitate the formation of hydroperoxy $(\mathrm{OOH})$ species, which is a crucial intermediate in the water oxidation process. ${ }^{87,88}$ A similar MOF-74 based electrocatalyst had also been prepared by Zheng et al., using $\mathrm{Fe}-\mathrm{Ni}$ metal nodes. ${ }^{89}$ Interestingly, they discovered that the initial source of iron metal used for doping the Ni-MOF seemed to affect the catalytic performance of the MOF catalyst. ${ }^{90}$ A similar conclusion was also reached by Zhang et al. in their recent work, where they reported a series of Fe doped Ni-MOFs employing different Fe ion sources. In another work by Wan et al., morphologydependent catalytic activity of a MOF was observed, where a 3D-flower like structure was formed out of MOF nanosheeets. ${ }^{91}$

The use of two-dimensional (2D) MOFs as catalysts has an added advantage of more exposed active sites and better ion or mass transport across the layers. Owing to these benefits, the use of MOF nanosheets as an OER catalyst is slowly gaining popularity among researchers. In a recent work, Li et al. prepared nanosheets of a two-dimensional (2D)-MOF $\left(\mathrm{Co}_{x} \mathrm{Fe}-\mathrm{MOF}\right)$, isostructural to $\mathrm{Co}_{2}(\mathrm{OH})_{2} \mathrm{BDC}$ MOF. ${ }^{92}$ The optimized catalyst $\mathrm{Co}_{3} \mathrm{Fe}-$ MOF had better efficiency than its single-metal counterparts, CoMOF and Fe-MOF. And the prime reason behind such an effect was the higher electrochemically active surface area (ECSA) of the mixed-node MOF than the rest. Another 2D CoFe-MOF was reported by Xu et al. as an electrocatalyst for the OER. ${ }^{93}$ But what was more important in this work was the synthetic strategy behind the formation of highly crystalline MOF nanosheets with a network of micro- and mesopores. Its synthetic procedure comprised of a two-step - ultrasound-assisted synthesis of MOF 


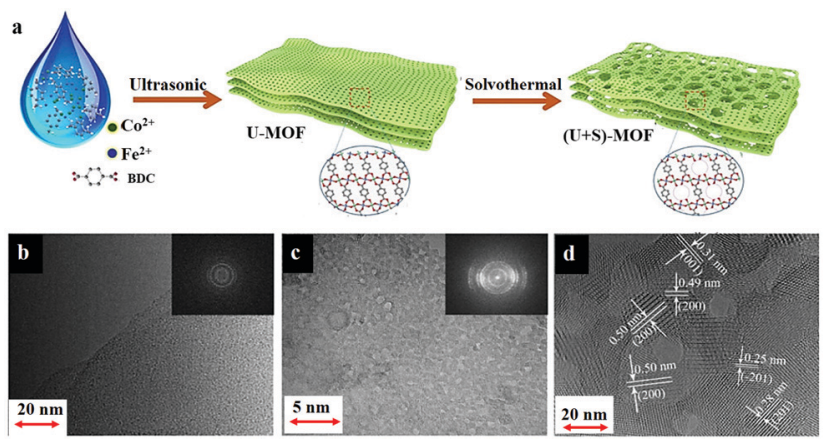

Fig. 2 (a) Schematic illustration of the two-step synthesis of hierarchical 2D CoFe-MOFs, involving ultrasound-assisted (U) synthesis (Step I) followed by the solvothermal (S) treatment (Step II). It also shows the structural evolution of the MOF nanosheets in the process. Comparison of TEM images of the CoFe-MOFs prepared by (b) only ultrasonic synthesis and (c) the two-step synthesis, with the inset of the SEAD patterns, respectively. (d) HRTEM image of $(U+S)$-CoFe-MOF, showing the crystalline structure and hierarchical pores. Adapted with permission from (ref. 93). Copyright (2019) American Chemical Society.

nanosheets, followed by solvothermal treatment (Fig. 2). The material thus formed had hierarchical porosity with a higher number of exposed metal sites, which is one of the most desirable properties of any MOF-based OER catalyst. Apart from using different combinations of just two metal ions, researchers have even gone for preparing trimetallic MOFs for catalysing the OER. In one such work, Bai et al. prepared a hierarchical coordination polymer film employing $\mathrm{Co}$, $\mathrm{Ni}$, and $\mathrm{Fe}$ metal sources. ${ }^{94}$ The novelty of the work lies in the synthetic procedure, where they had used a spray-assisted miscible liquid-liquid interface (MLLI) strategy, which led to the formation of films having a 3D hierarchical network structure. ${ }^{95}$ Another such work focusing on the preparation of trimetallic-MOFs having a hierarchical foam-like architecture, was reported by Qian et al. In this work, the authors presented a mild one-pot room temperature synthesis for the large-scale synthesis of the NiCoFe-based MOF nanofoam (denoted as $\left(\mathrm{Ni}_{2} \mathrm{Co}_{1}\right)_{1-x} \mathrm{Fe}_{x}$-MOF-NF).

MOFs with mixed-nodes are in general found to perform better than their single-metal counterparts, as an OER electrocatalyst, owing to the enhanced charge-transfer kinetics and their increased electrochemically active surface area (ECSA). Table 1 compares the OER activities of metal node engineered MOFs.

\section{1c. Linker engineered MOFs for OER catalysis}

Even though there are a lot of studies on the effect of incorporation of different metal ions in a MOF on its OER catalytic activity, much less has been explored on the effect of ligand modification. The early examples of ligand modification can be found in the works of Gong et al., where they prepared MOFs with the same metal ion and linker ligands, but different coligands. ${ }^{96,97}$ And interestingly, certain differences in the electrochemical activity of the sister MOFs were observed, due to the differences in their co-ligands and electrochemical environment.

In another work, Johnson et al. by means of a post-synthetic ligand exchange method incorporated a molecular OER catalyst
Table 1 Comparison of the OER activities of metal node engineered MOFs

\begin{tabular}{|c|c|c|c|}
\hline Catalyst & $\begin{array}{l}\text { Overpotential } \eta_{x}(\mathrm{mV}) \\
x=\text { current density } \\
\left.(\mathrm{mA} \mathrm{cm})^{-2}\right)\end{array}$ & $\mathrm{pH}$ & Ref. \\
\hline $\mathrm{Co}_{2}$-MOF@Nafion & $\eta_{2}=460, \eta_{5}=537$ & 7 & 78 \\
\hline Compound 1 & $\eta_{1}=257$ & 7 & 79 \\
\hline CoCd-BNN & $\eta_{1}=353$ & 13 & 84 \\
\hline CoFe-PYZ & $\eta_{10}=300$ & 13 & 85 \\
\hline NiCo-PYZ & $\eta_{10}=362$ & 13 & 85 \\
\hline NiFe-PYZ & $\eta_{10}=560$ & 13 & 85 \\
\hline $\mathrm{Co}_{0.6} \mathrm{Fe}_{0.4}$-MOF-74 & $\eta_{10}=280$ & 14 & 86 \\
\hline $\mathrm{Co}_{3} \mathrm{Fe}-\mathrm{MOF}$ & $\eta_{10}=280$ & 14 & 92 \\
\hline CoFe-MOF & $\eta_{10}=277$ & 14 & 93 \\
\hline FeNi-DOBDC-3 & $\eta_{50}=270, \eta_{100}=287$ & 14 & 84 \\
\hline UTSA-16 & $\eta_{10}=408$ & 14 & 80 \\
\hline $\mathrm{Fe}_{0.38} \mathrm{Ni}_{0.62}-\mathrm{MOF}$ & $\eta_{10}=190$ & 14 & 91 \\
\hline$(\mathrm{Ni2Co1})_{0.925} \mathrm{Fe}_{0.075}$-MOF-NF & $\eta_{10}=257$ & 14 & 95 \\
\hline $\mathrm{PCN}-\mathrm{Fe}_{2} \mathrm{Co}-\mathrm{Fe}_{2} \mathrm{Ni}$ & $\eta_{10}=271$ & 14 & 82 \\
\hline
\end{tabular}

$\left[\mathrm{Ru}(\mathrm{tpy})(\mathrm{dcbpy})\left(\mathrm{OH}_{2}\right)\right]\left(\mathrm{ClO}_{4}\right)_{2}$ (tpy $=2,2^{\prime}: 6^{\prime}, 2^{\prime \prime}$-terpyridine, dcbpy $=$ 2,2'-bipyridine-5, $5^{\prime}$-dicarboxylic acid) into a FTO-grown thin film of UiO-67 MOF, to give a MOF-based OER catalyst, UiO67$\left[\mathrm{RuOH}_{2}\right] @ \mathrm{FTO} .{ }^{44}$ Later, the same group designed another OER catalyst by means of post-synthetic ligand modification from MIL-101Cr MOF. ${ }^{98}$ In this case, a Ru(bda) (bda = 2,2' -bipyridine6,6'-dicarboxylate)-based molecular OER catalyst was very strategically placed into the pores of the MOF by means of anchoring it with the linker ligand of the concerned MOF. The catalyst thus formed in both cases, turned out to be a better OER catalyst than their respective Ru-based molecular catalyst complex. It is believed that the immobilization of the molecular catalyst and modified micro-environment around it, inside the MOF cavity, provided enough stability to it to perform as a better catalyst.

Xue et al. explored the possibility of combining both the strategies of using mixed-metal nodes as well as using mixed ligands to prepare a MOF-based OER catalyst. ${ }^{99}$ This heterogeneous MOF, defined as $\mathrm{A}_{2.7} \mathrm{~B}-\mathrm{MOF}-\mathrm{FeCo}_{1.6}$, was prepared by using two different linker ligands - terephthalic acid (A) and 2-aminoterephthalic acid (B). In the following year, 2019, another mixed ligand MOF was reported by Han et al., prepared by employing mixed organic ligands including rigid 1,5-bi(imidazolyl)anthracene and dibenzobarrelene skeletons based dicarboxylic acid. ${ }^{100}$

In another piece of work, a group of researchers studied the role of different anionic ligands in a bimetallic MOF derived OER catalyst, in tuning the electronic environment around the active catalytic center. ${ }^{101}$ Ligand modification has also been reported to influence the transformation of MOFs to $\mathrm{Co}(\mathrm{O}) \mathrm{OH}$ phase during an electrocatalytic process. ${ }^{102}$ And a systematic study of the same can help us to understand and control this transformation process in order to prepare better OER catalysts.

\section{Guests in MOFs for electrocatalytic OER}

An important feature of the MOF architecture is its pores and exceptionally high porous volume. If the pores or cages of MOFs can be functionalized by confining any catalytically active 


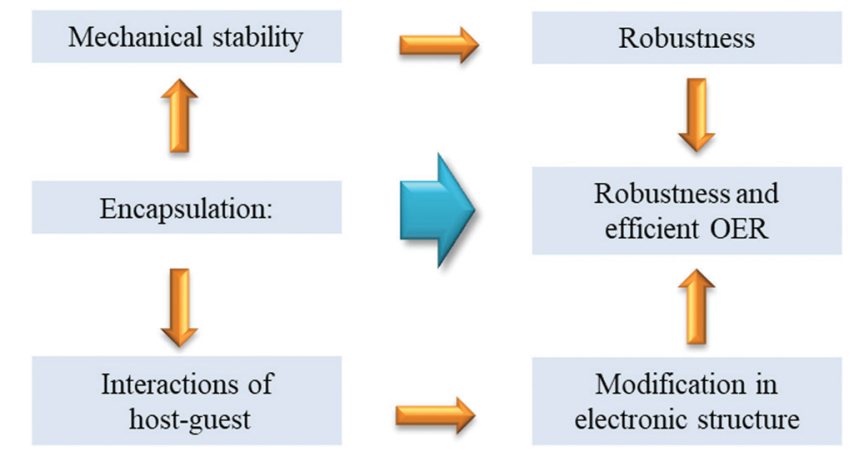

Scheme 4 Benefits of guest encapsulation in MOFs for OER catalyst designing.

species inside it such that it will not be able to leach out of the pore/cage due to geometrical constraints, or due to interaction with the internal surface of the MOF, it can be termed as 'activation by means of encapsulation' (Scheme 4).

Although this fundamental approach of preparing heterogeneous functional materials is well-studied for various porous materials, e.g., zeolites, it is not yet fully explored to design MOF-based OER catalysts. Reports of organic substrate oxidation based on Metal@MOF ${ }^{103,104}$ and Molecule@MOF ${ }^{105}$ are more frequent than similar OER catalysts. For designing a guest@MOF OER catalyst, the choice of the MOF and the guest species is crucial and depends on several factors. One of the most important factors is the size consideration, i.e., the size of the guest should be small enough so that it fits well inside the cavity of the MOF but it should be big enough not to leach out of the windows of the cavity. The host MOF in this case might actively participate in the catalysis, or may just act as a storehouse for the catalyst. But whichever the case may be, it has been found in several reports that the MOF framework plays some role in modifying the electronic structure of the guest molecule and thus, may also influence its catalytic activity. Guo et al. reported excellent OER activity by Pt-loaded Co and NiPrussian Blue analogous (PBA) MOFs. ${ }^{106}$ Here, the MOFs had an intrinsic OER catalytic property, which was considerably benefitted by the loading of Pt nanoparticles. For both PBAs, the oxidation state of $\mathrm{Co} / \mathrm{Ni}$ was slightly increased due to the incorporation of $\mathrm{Pt}$, which acted as an electronegative metal source and stabilized the active metal center in its partially increased oxidation state. With the loading of Pt nanoparticles, the overpotential requirement came down for Co/Ni-PBA and was reported to be comparable with the benchmark electrocatalyst $\mathrm{RuO}_{2}$. The Pt nanoparticle loading resulted in (a) lowering of charge transfer resistance, (b) increase in the number of active sites and (c) stability of $\mathrm{Co} / \mathrm{Ni}$ in a higher oxidation state; all of which contributed to the better performance of the modified MOF-based catalysts (Co-Pt-PB and Ni-Pt-PB). In a different approach, Nepal and Das encapsulated a high-valent dimeric Mn complex, MnTD ([(terpy)Mn $\left.(\mu-\mathrm{O})_{2} \mathrm{Mn}\right][(\text { terpy })]^{3+}$; terpy: 2,2':6,2'-terpyridine) inside the cavities of MIL$101(\mathrm{Cr}){ }^{107}$ The composite thus formed was named as MnTD $\subset$ MIL-101(Cr). The $\operatorname{Mn}(\mu-\mathrm{O})_{2} \mathrm{Mn}$ complex as such is active towards OER catalysis but is not stable under the high oxidizing potential of the process. It undergoes oxidative polymerization through an intermolecular reaction pathway and hence loses its activity. By means of encapsulation of the molecule inside the single cages of the MOF, a physical separation was introduced between each unit of $\mathrm{Mn}(\mu-\mathrm{O})_{2} \mathrm{Mn}$; which prevented its oxidative dimerization. The host-guest composite material was then found to be a promising photocatalyst for the OER.

A similar "ship-in-a-bottle" approach was also explored by our group to prepare a MOF-based OER electrocatalyst. Manna et al. by means of solvothermal synthesis trapped a Co-complex $\left[\mathrm{Co}\left(\mathrm{H}_{2} \mathrm{O}\right)_{4}(\mathrm{DMF})_{2}\right]_{2}$ inside the cavity of a flexible ligand contain-

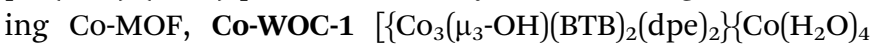
$\left.\left.(\mathrm{DMF})_{2}\right\}_{0.5}\right]_{n} \cdot n \mathrm{H}_{2} \mathrm{O} \quad\left(\mathrm{H}_{3} \mathrm{BTB}=1,3,5\right.$-benzenetribenzoic acid; dpe $=1,2$-di(4-pyridyl)ethylene) ${ }^{108}$ As a result of confinement, the active unit $\left[\mathrm{Co}\left(\mathrm{H}_{2} \mathrm{O}\right)_{4}(\mathrm{DMF})_{2}\right]_{2}$ could not leach out of the MOF during the electrochemical operational conditions (Fig. 3a). The observed TOF was $0.05 \mathrm{~s}^{-1}$, while $\eta_{1}$ was $390 \mathrm{mV}$. The importance of this heterogenous catalyst lies in the enhancement of stability of the trapped $\left[\mathrm{Co}\left(\mathrm{H}_{2} \mathrm{O}\right)_{4}(\mathrm{DMF})_{2}\right]_{2}$ unit inside the MOF cavity. The lack of scope of structural rearrangement of the Co-complex to develop an extended structure while being inside the MOF crystal, seems to play a crucial role behind the high stability of the host-guest electrocatalyst. ${ }^{103}$

In another work from our group, a host-guest composite (POM@ZIF-8) was prepared by means of in situ encapsulation of an unsubstituted Keggin polyoxometalate (POM), i.e., $\mathrm{K}_{6}\left[\mathrm{CoW}_{12} \mathrm{O}_{40}\right]$ (guest) inside ZIF-8 (host) (Fig. 3b). ${ }^{109}$ Due to structural constraints of Keggin POM, it was not expected to act as an OER catalyst, and this is what was found from the electrochemical analysis as well (Fig. 3c), which suggests a complete breakdown of its structure. However, the POM@ZIF-8 composite could perform electrocatalytic OER for a prolonged time. This encapsulation strategy triggered the activation (as probed by XPS analysis) and stabilization (proved by long term electrochemical experiments, XRD, ICP, FESEM analysis, etc.) of the POM towards OER catalysis. A shift in electron density from the electron rich internal surface of ZIF-8 to the low lying ' $W$ ' centred LUMOs of the POM were found to be crucial. POM@ZIF-8 could perform electrocatalytic OER with high efficiency $\left(\mathrm{TOF}=10.8 \mathrm{~s}^{-1}\right.$ ) and high stability in neutral $\mathrm{pH}$, having a satisfactory faradaic efficiency ( $\geq 95 \%)$. This is one of the highest TOFs reported for any heterogeneous electrocatalyst performing the OER in neutral $\mathrm{pH}$. The inherent resistive nature of ZIF-8 was responsible for the high overpotential requirement $\left(\eta_{1}=780 \mathrm{mV}\right)$.

A similar in situ encapsulation of a catalytically active Keggin POM inside the cavity of ZIF-8 and ZIF-67 was explored by Abdelkader-Fernández et al. to study the effect of the encapsulation on their OER catalysis (Fig. 3d) ${ }^{110}$ It was found that the OER activity of the host-guest composite POM@ZIF-67 was better than the POM or ZIF-67 as such; while no notable enhancement was observed in the case of the POM@ZIF-8. A comparison of these systems helped the authors to understand the two ways in which the encapsulation helped the OER 
(a)

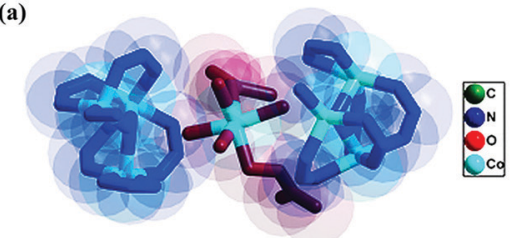

(b)

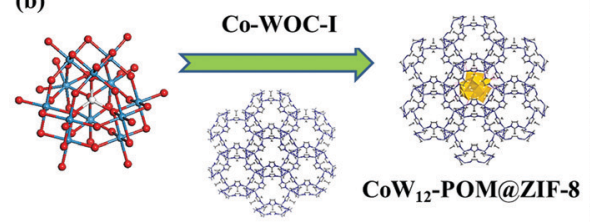

(c)

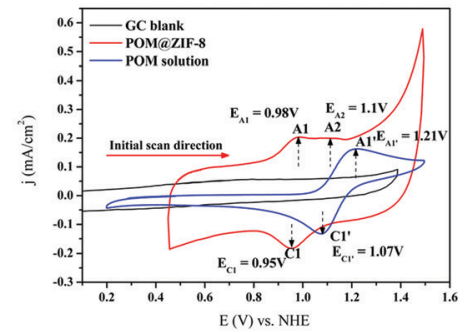

(d)
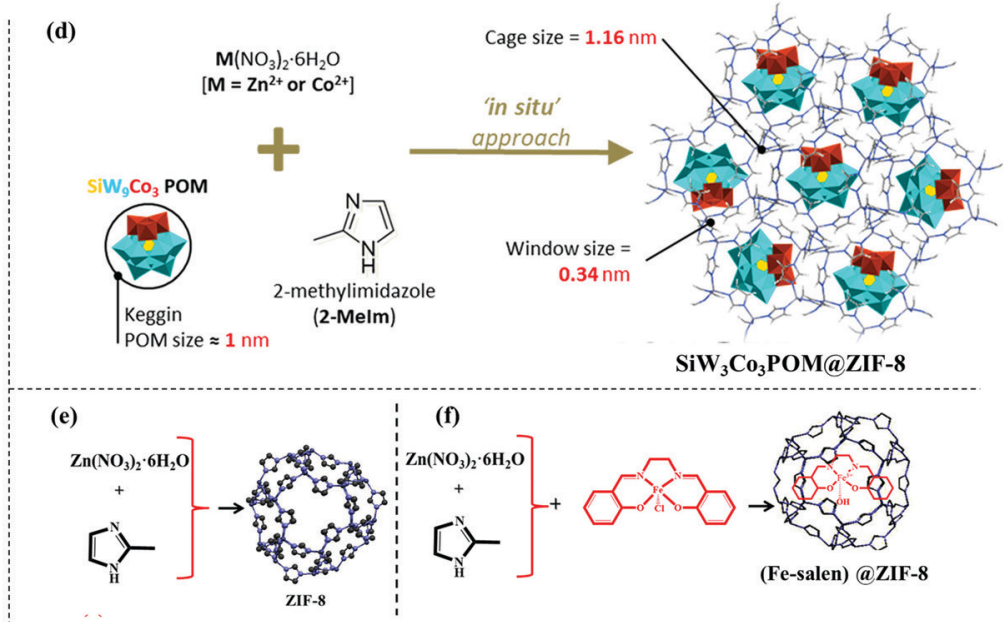

(g)

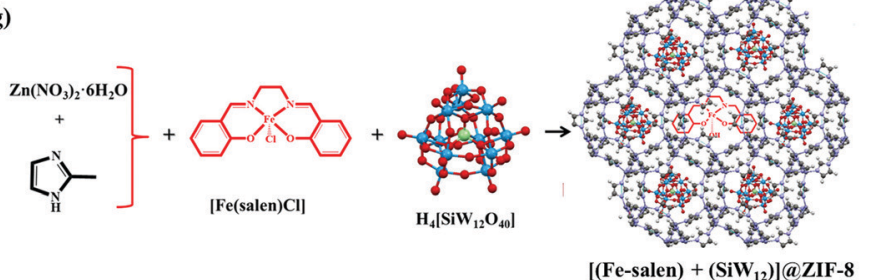

Fig. 3 (a) Active site structure of Co-WOC-1. Adapted with permission from (ref. 108). Copyright (2016) John Wiley \& Sons. (b) In situ encapsulation of $\left[\mathrm{CoW}_{12} \mathrm{O}_{40}\right]^{6-}$ Keggin inside the $\mathrm{ZIF}-8$ cavity; (c) cyclic voltammograms of POM(aZIF-8 and $\mathrm{K}_{6}\left[\mathrm{CoW}_{12} \mathrm{O}_{40}\right.$ ] recorded in pH $20.1 \mathrm{M} \mathrm{KCl}$; reprinted with permission from (ref. 109). Copyright (2018) John Wiley \& Sons. (d) Synthetic protocol of $\mathrm{SiW}_{9} \mathrm{Co}_{3} \mathrm{POM}(\mathrm{ZIF}-8$, reprinted with permission from (ref. 110). Copyright (2020) American Chemical Society. (e), (f), (g) Synthetic protocol of ZIF-8, Fe(salen)@ZIF-8 and [(Fe-salen) + (SiW 12$)] @ Z I F-8$, respectively. Adapted with permission from (ref. 111). Copyright (2020) American Chemical Society.

catalysis of the composite, i.e., (a) synergistic interaction between the POM and the MOF inner surface and (b) an increase in defects in the MOF as a result of the encapsulation. ${ }^{105}$

Recently, another OER catalyst based on a host-guest system was prepared by us using ZIF-8 as a host and [Fe(salen)] $\mathrm{Cl}$ as the guest species (Fig. 3e and f). ${ }^{111}$ The guest as such is unstable towards electrochemical OER but was found to function as a stable OER catalyst after encapsulation. This was the first report of catalytic OER activity by Fe-salen. Furthermore, a Keggin POM $\left[\mathrm{SiW}_{12} \mathrm{O}_{40}\right]^{4-}$ was co-encapsulated in the cages and also was grown on the surface of ZIF-8 nanocrystals with an aim to increase the charge transfer efficiency. This strategy was reported to perform electrocatalytic OER with a high TOF of $\sim 5 \mathrm{~s}^{-1}$ and comparatively low overpotential requirements. A similar approach was also found to be helpful to prepare another electrocatalyst for the OER using a porphyrin molecule, i.e., Co-tetramethoxy phenylporphyrin (CoTMPP) as a guest and ZIF- 8 as a host material. The catalyst thus prepared, i.e., CTMZ-8 was found to be an active OER catalyst in a wide pH window ranging from neutral to acidic. The above-mentioned catalyst was reported to perform the OER with a TOF of $\sim 2.7 \mathrm{~s}^{-1}$ and an overpotential requirement of $387.4 \mathrm{mV}$ to achieve a current density of $1 \mathrm{~mA} \mathrm{~cm}{ }^{-2} \cdot{ }^{112}$ All these examples show that guest@MOF can be a very promising approach to design a MOF based electrocatalyst for the OER, because it can act as a bicomponent system in synergistic interaction with each other, where each component has its own role in the overall catalysis process. The design strategy of guest@MOF composites involves plenty of scope of in situ and ex situ modifications, which may lead to unprecedented catalytic activity and stability.

\section{MOF-based hybrid composites}

From earlier sections, it is evident that there are a wide variety of MOFs for electrocatalytic water oxidation reactions. But, because of their poor conductivity and comparatively low stability limits, their applicability for OER catalysis under harsh experimental conditions like varying $\mathrm{pH}$ and high applied voltages is limited. To circumvent these disadvantages, many research groups have been working on designing and fabricating hybrid electrocatalysts by combining MOFs with other conducting materials, e.g., conducting organic polymers, graphene, carbon nanotubes (CNTs), MXene type metal carbides/ nitrides, metal and metal oxides, etc.

\section{3a. Organic polymers/MOF hybrids}

There are a few examples related to preparation of hybrids with organic polymers and MOFs, e.g., PANI/MOF, PABA/MOF etc. $($ PANI $=$ polyaniline; PABA $=\operatorname{poly}(3$-aminobenzoic acid $))$ for applications in diverse fields including catalysis. ${ }^{113-116}$ In most of these cases, the polymer played an important role to enhance the electronic conductivity of the composite. Though there are many such examples of polymer/MOF hybrids, systems dealing 
with electrocatalytic OER are not very common. Dang et al. reported an organic polymer supported MOF, which had been employed for electrocatalytic OER reactions. ${ }^{117}$ A bi-metallic (CoNi-NDC) MOF was solvothermally grown on to a polyaniline (PANI) layer deposited onto a nickel foam (NF) current collector to yield CoNi-NDC/PANI-NF electrode. The hybrid electrode could behave as a durable electrocatalyst for the OER with low overpotential $\left(\eta_{10}=353 \mathrm{mV}\right)$ requirement and also followed a fast kinetics (Tafel slope $=73.3 \mathrm{mV}$ decade $^{-1}$ ). The higher activity was attributed to the presence of conductive polymer, which helped in effective charge transfer from MOF to NF. This example certainly proves that there is a lot of scope in exploring conductive organic polymer/MOF hybrid based OER electrocatalysts.

\section{3b. Conducting carbon material/MOF hybrids}

Owing to their high surface area and oxygen rich surfaces, conducting carbon-based materials like graphene oxide (GO) and CNTs are anticipated to be ideal candidates to accompany MOFs resulting in hybrid composites. As improving charge transfer ability of the MOF composite would increase the catalytic activity, Sohrabi et al. ${ }^{118}$ fabricated a 3D network of PCN-224/multiwalled carbon nanotube (PCN-224/MWCNT, PCN = porous coordination network) comprising $3 \mathrm{D}$ nanochannels. It was presumed that MWCNTs would contribute towards enhancing the conductivity. Whereas, the rationale behind selection of $\mathrm{PCN}-224$ was the presence of robust $\mathrm{Zr}_{6}$ nodes. On the other hand, a metalloporphyrin, tetrakis(4-carboxyphenyl)porphyrin-Co ${ }^{\text {II }}$ (CoTCPP) linker with a coordinatively unsaturated and catalytically active cobalt center would make the composite a good candidate towards the OER.

Besides CNT/MOF composites, there are ample reports on graphene/MOF hybrids, where graphene not only acts as a conducting support to grow the MOF but also prevents aggregation. A report from Xie et al. ${ }^{119}$ illustrated excellent catalytic OER by $10 \mathrm{mg}$ 3D graphene (Gr) loaded Gr/Ni-MOF composite. The enhanced activity was attributed to fully exposed, ultrasonically dispersed, and strongly bound Ni-MOF along with synergistic interaction between the MOF and graphene.

In addition, there are some interesting examples, where these $1 \mathrm{D}$ and $2 \mathrm{D}$ carbons act as struts and penetrate the MOF crystal lattices. Oxy groups of the carbon material directly take part in the formation of the metal coordination sphere and strengthen the composite structure. Apparently, this strut property of carbons not only stabilizes the composite even in the most harsh reaction conditions, taking advantage of effective communication between active metal centers and conducting medium, but also enhances catalytic activity. Jahan et al. ${ }^{120}$ solvothermally fabricated a potent tri-functional (effective catalyst for HER, OER and ORR) electroctalyst, grapheneoxide (GO)/MOF composite. Later on, a strong, durable, and highly active Co-MOF@CNTs bifunctional catalyst was grown following a self-assembly strategy by Fang et al., ${ }^{121}$ where the CNTs acted as struts and were inserted into the MOF crystals. The augmented activity of the composite was ascribed to the synergistic interaction between the metal, ligand, and CNT struts.

\section{3c. MXene/MOF hybrids}

MXenes are a class of transition metal carbides, nitrides and carbonitrides and are relatively young members in the $2 \mathrm{D}$ conductive layered materials. These are generally few-atomthick 2D layers, and due to their flexible nature, excellent conductivity and ease of processability, these materials have soon been found to be useful in various applications. They are generally formulated as $\mathrm{M}_{n+1} \mathrm{X}_{n} \mathrm{~T}_{x}$ ( $\mathrm{M}=$ transition metal, $\mathrm{X}=\mathrm{C}$ and/or $\mathrm{N}, \mathrm{T}_{x}=$ surface functional groups like $-\mathrm{O},-\mathrm{OH}$, and/or $-\mathrm{F}$, etc.) and because of their unique atomic arrangements, they are intrinsically conductive in nature and are among the best candidates in providing conductive supports/surfaces to fabricate organic-inorganic composites. There are a few instances, where MXenes were found to act as a conductive support for MOF-based OER catalysts, where a synergistic interaction between the two enhanced the catalytic performance of the composite. Zhao et al. ${ }^{122}$ fabricated a CoBDC/ $\mathrm{Ti}_{3} \mathrm{C}_{2} \mathrm{~T}_{x}(\mathrm{BDC}=$ 1,4-benzene dicarboxylate) hybrid material, adapting an interdiffusion reaction-assisted process, where CoBDC was grown on 2D nanosheets of MXene. As a result of their synergistic interactions between the MOF and the $\mathrm{Ti}_{3} \mathrm{C}_{2} \mathrm{~T}_{x}$, the hybrid could behave as a good electrocatalyst for the OER with low overpotential $\left(\eta_{10}=410 \mathrm{mV}\right)$ requirement and also followed a fast kinetics (Tafel slope $=48.2 \mathrm{mV}$ decade $^{-1}$ ). Similarly, a bimetallic MOF (CoNi-ZIF-67) and MXene based hybrid electrocatalytic OER catalyst (CoNi-ZIF-67@ $\mathrm{Ti}_{3} \mathrm{C}_{2} \mathrm{~T}_{x}$ ) was reported by Wen et al. ${ }^{123}$ adopting a coprecipitation technique. Systematic electrocatalysis examinations revealed that, the generated hybrid exhibits better catalytic activity than the standard $\mathrm{IrO}_{2}$ and the MOF itself. The enhancement in electrocatalytic activity was attributed to the three-fold benefits, that is (a) synergistic effects resulting into enhanced conductivity, (b) increased oxidation states of $\mathrm{Co}$ and $\mathrm{Ni}$ on the introduction of MXene to MOF, and (c) smaller MOF particle size along with enhanced electrochemically active surface area (EASA), etc.

\section{3d. Metal chalcogen/MOF hybrids}

Generally metal oxide nanoparticles (MNPs) are catalytically active towards electrocatalytic OER. ${ }^{124,125}$ But, owing to their small size, large surface area and surface energy they are prone to form aggregates and reduce their efficiency on their application for a prolonged period. ${ }^{48}$ In this context, MOFs not only provide them support but also, in suitable conditions, they can form core (MNP)-shell (MOF) type composites. Additionally, the resultant composite, being a multimetallic system with incoming metal entities, act synergistically and enhance the overall catalytic efficiency.

Evidently in one of their reports, Wang et al. ${ }^{126}$ have fabricated a Ni-BDC-MOF shell protected $\mathrm{Ni}_{3} \mathrm{~S}_{2}$ /NiS hollow nanoparticle (NP) based catalytic system for OER applications. The MOF was grown on the preformed MNPs solvothermally (Scheme 5). The composite showed improved activity compared to that of its individual components and even outperformed the $\mathrm{Ru}_{2} \mathrm{O}$ reference. The hydrophilicity and coordinative unsaturation of the Ni-BDC shell and highly porous and conducting $\mathrm{Ni}_{3} \mathrm{~S}_{2} / \mathrm{NiS}$ 


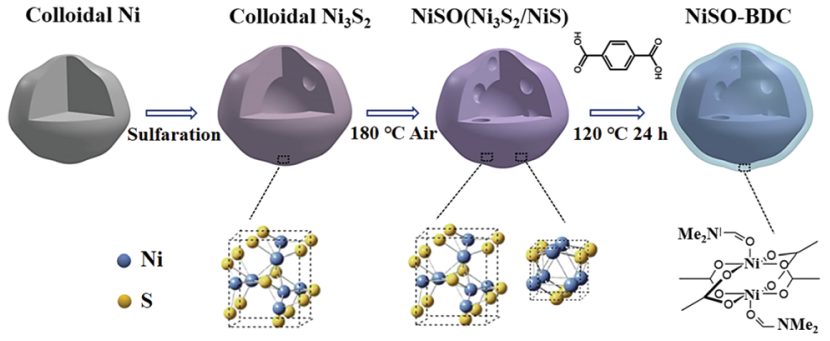

Scheme 5 Schematic illustration of the preparation of $\mathrm{Ni}$-BDC-modified $\mathrm{Ni}_{3} \mathrm{~S}_{2} / \mathrm{NiS}$ (NiSO-BDC) hollow nanoparticles. Reprinted with permission from (ref. 126). Copyright (2019) American Chemical Society.

core with strongly coupled interface contributed in enhancing the catalytic activity along with observed prolonged durability (notable activity for $15 \mathrm{~h}$ ).

MOFs can also act as a support to toggle MNPs and strategies have been developed for loading of MNPs onto MOF particles' surface. Gao et al. ${ }^{127}$ systematically loaded ultralow quantities of $\mathrm{Fe}_{2} \mathrm{O}_{3}$ particles on to the MOF particle surface in controllable ratios. They reproduced the Ni-MOF-74 containing linker with uncoordinating hydroxyl groups. Following unique and fast "phenol-iron" surface reactions, $\mathrm{Fe}_{2} \mathrm{O}_{3}$ particles were attached on to the surfaces of Ni-MOF-74 particles in different $\%$ amounts. Upon careful electrochemical analysis, the optimized OER catalyst was found to be $0.6 \mathrm{wt} \% \mathrm{Fe}_{2} \mathrm{O}_{3} @ \mathrm{Ni}-\mathrm{MOF}-74$ composite.

In a different approach, Zhang et al. ${ }^{128}$ exfoliated inorganic $\mathrm{CoFeO}_{x}$ nanoparticle embedded polycobalt benzimidazole monolayered nanosheets (M-PCBN) out of PCB (polycobalt benzimidazole) analogous framework. Systematic structural characterization revealed that MNP-MOF interfacial Co displays higher valency with changed $3 \mathrm{~d}$ electronic configuration compared to $\mathrm{CoN}_{4}$ from the framework part and was correlated to the enhanced activity, which was also supported by DFT calculations. Though these materials are broadly classified into different categories for convenience of the reader, there are a few isolated cases which stay in the borderline of these individual classes. One such instance of a three component system was reported by Srinivas et al., where $\mathrm{FeNi}_{3}-\mathrm{Fe}_{3} \mathrm{O}_{4} \mathrm{NPs}$ were anchored on to $\mathrm{NiFe}_{x}$-MOF nanosheets and the CNT matrix $\left(\mathrm{FeNi}_{3}-\mathrm{Fe}_{3} \mathrm{O}_{4} \mathrm{NPs} / \mathrm{MOF}-\mathrm{CNT}\right)$. Owing to synergistic interactions among all composite components, it showed excellent overall water splitting activity that surpassed the performance of commercially available electrocatalysts $\left(\mathrm{Pt} / \mathrm{C}\right.$ and $\left.\mathrm{RuO}_{2}\right) .{ }^{129}$

\section{3e. Metal/metal oxide supported surface-coordinated metal-organic framework thin films (SURMOFs)}

Fabrication of MOF-based composites with conducting substrates, like carbon materials and/or MNPs, has opened up new aspects of designing MOF-based OER catalysts but they are coming up with their own challenges. In the majority of cases, the active catalysts, either being drop casted on to the electrode surface in its native dilute colloidal form (which may leach out) or with binders like Nafion (which insulates and dilutes active sample besides masking pores from reactants), lead to hampering of the efficiency of the catalyst. It showed the dire need of fabricating binder free electrode materials which strongly bind to current collectors with uniform arrangement to minimize issues, related to particle grain boundary. There have been different methods, like direct growth/deposition, layer-by-layer deposition and electrochemical deposition on conducting substrates, like FTO, metal plates and/or porous metal foams even occasionally on to GC active surfaces etc.

Working in this direction, a Prussian blue-type cobalt hexacyanoferrate (CoHCF) was fabricated on an FTO (fluoride-doped tin oxide) coated glass electrode by Pintado et al., ${ }^{130}$ following electrochemical methods. The stepwise procedure involves electroplating of a thin layer of cobalt metal which was later treated electrochemically in the presence of $\left[\mathrm{Fe}^{\mathrm{III}}(\mathrm{CN})_{6}\right]^{3-}$ leading to a Co-Fe Prussian blue modified FTO electrode. The modified electrode could hold its activity for an extended period (even for weeks), without considerable drop in its efficiency. ${ }^{117}$

Owing to the ability to maximize accessible catalytic sites and decreased ion transfer distances favoring effective electrical conductivity, it is found to be advantageous to use metal foam type porous conducting substrates to grow/deposit MOFs. The intrinsic spongy texture of these foams allows the fabrication of more efficient and hierarchical porous materials in combination with MOFs with catalytically active sites. Zhao et al. supplemented this fact that thin film-based electrodes exhibit superior electrocatalytic activity over electrodes with bulk material; and films deposited on porous materials show better OER activity over nonporous electrodes. ${ }^{34}$ Their studies concluded that the bulk MOF possessing GC showed inferior activity in comparison to GC with ultrathin MOF nanosheets of NiCo-MOF (NiCo-UMOFN) (Fig. 4a). On the other hand, NiCo-UMOFN deposited $\mathrm{Cu}$ foam exhibited better catalytic activity over GC.

There are ample examples, where metal foams can act as sacrificial metal substrates to fabricate mixed metal MOF thin films. Ye et al. demonstrated that two dimensional nanosheet arrays of Co/Ni-based mixed MOF (NiCo-9AC-AD) with controllable and measured composition of $\mathrm{Ni}_{0.3} \mathrm{Co}_{0.7}-9 \mathrm{AC}-\mathrm{AD} / \mathrm{NF}$ were grown on nickel foam (NF) following a simple solvothermal procedure. ${ }^{131}$ Systematic electrocatalytic analysis concluded that, the presence of ultrathin nano sheets and synergic interactions between $\mathrm{Ni} / \mathrm{Co}$ catalytic sites resulted in the excellent OER and HER activity. Similarly, Ling et al. ${ }^{132}$ fabricated $\mathrm{Fe}_{2} \mathrm{Ni}$ $\mathrm{MOF} / \mathrm{NF}$ ( $\mathrm{Fe}_{2} \mathrm{Ni}-\mathrm{MIL}-88 \mathrm{~B}$ MOF on nickel foam) and explained

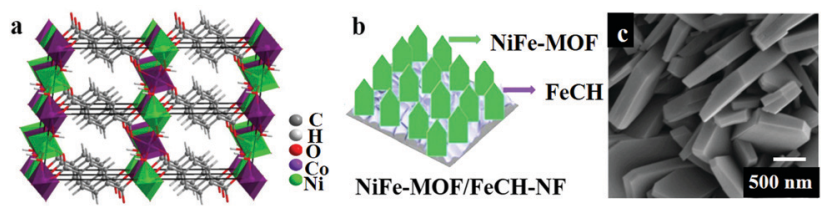

Fig. 4 (a) The overall crystal structure of ultrathin MOF nanosheets of NiCo-MOF (NiCo-UMOFNs), with three coordination structural layers. Reprinted with permission from (ref. 34). Copyright (2016) Springer Nature. (b) A representative diagram of the fabrication of NiFe-MOF/FeCH-NF. (c) SEM image of NiFe-MOF/FeCH-NF. Adapted with permission from ref. 133 with the permission of The Royal Society of Chemistry. 
Table 2 Comparison for the OER activities of MOF-based hybrid composites

\begin{tabular}{|c|c|c|c|}
\hline Catalyst & $\begin{array}{l}\text { Overpotential } \eta_{x}(\mathrm{mV}) \\
x=\text { current density } \\
\left.(\mathrm{mA} \mathrm{cm})^{-2}\right)\end{array}$ & $\mathrm{pH}$ & Ref. \\
\hline (GO 8 wt\%) Cu-MOF & $\eta_{2}=110$ & $<1$ & 120 \\
\hline CoHCF & $\eta_{1}=400$ & 7 & 130 \\
\hline PCN-224/MWCNT & $\eta_{2}=510$ & 9.2 & 118 \\
\hline 3D Gr/Ni-MOF & $\eta_{10}=370$ & 13 & 119 \\
\hline Co-MOF@CNTs (5 wt\%) & $\eta_{57}=440$ & 14 & 121 \\
\hline NiSO-BDC & $\eta_{10}=298$ & 14 & 126 \\
\hline 0.6 wt $\% \mathrm{Fe}_{2} \mathrm{O}_{3} @ N i-M O F-74$ & $\eta_{10}=264$ & 14 & 127 \\
\hline M-PCBN & $\eta_{10}=232$ & 14 & 128 \\
\hline $\mathrm{FeNi}_{3}-\mathrm{Fe}_{3} \mathrm{O}_{4}$ NPs/MOF-CNT & $\eta_{10}=234$ & 14 & 129 \\
\hline NiCo-UMOFNs/Cu foam & $\eta_{10}=189$ & 14 & 34 \\
\hline $\mathrm{Ni}_{0.3} \mathrm{Co}_{0.7}-9 \mathrm{AC}-\mathrm{AD} / \mathrm{NF}$ & $\eta_{100}=350$ & 14 & 131 \\
\hline $\mathrm{Fe}_{2} \mathrm{Ni} \mathrm{MOF} / \mathrm{NF}$ & $\eta_{10}=222$ & 14 & 132 \\
\hline NiFe-MOF/FeCH-NF & $\eta_{10}=200$ & 14 & 133 \\
\hline CoNi-MOFNA & $\eta_{10}=215$ & 14 & 134 \\
\hline Pt QDs@Fe-MOF/NF & $\eta_{100}=191$ & 14 & 135 \\
\hline
\end{tabular}

its superior electrocatalytic activity. In another instance, NF (nickel foam) was demonstrated to act as a support for iron carbonate hydroxide nanosheets ( $\mathrm{FeCH} \mathrm{NS}$ ) which was the sacrificial template for Fe/Ni MOF fabrication (Fig. $4 \mathrm{~b}$ and c). ${ }^{133}$

The sacrificial template not only releases iron ions for MOF fabrication, it also slows down the release of $\mathrm{Ni}$ ions, and in turn, controls the composition of the thin film. Additionally, unreacted $\mathrm{FeCH}$ NS also contributes synergistically in boosting the catalytic activity of the resultant electrode material (NiFeMOF/FeCH-NF). Likewise, there are also bimetallic alloys employed for metal foam supported MOF fabrication.

In a recent report, Huang et al. have shown that CoNi MOF nano arrays (CoNi-MOFNA) can be grown on a CoNi alloy following an in situ self-dissociation-assembly (SDA) synthetic strategy. ${ }^{134}$ The fabricated material with ultrathin nano arrays, constructed of a synergistically acting bimetallic system having unsaturated coordination sites, shows outstanding OER activity compared to commercially available $\mathrm{RuO}_{2}$ standard. Besides, Ye et al. reported a borderline multicomponent Fe-MOF/NF composite with Pt quantum dots (Pt QDs) incorporated into Fe-MOF nanosheet arrays/shells, that were supported on a nickel foam (Pt QDs@Fe-MOF/NF). This composite, on thorough catalytic analysis, showed excellent water splitting activity with good stability which was attributed to enhanced electron transfer rate by porous Pt QDs@MOF core-shell structure. ${ }^{135}$ Table 2 compares the OER activities of MOF-based hybrid composites.

\section{Hybrid materials derived by pyrolysis of MOFs}

As discussed in earlier sections, designing and structural engineering of MOFs and their hybrid composites have been definitely one of the finest approaches in fabricating MOFbased electrocatalysts for OER applications. However, the applicability of MOFs is not only limited to their native or hybrid forms. MOF derivatization via thermal annealing and graphitization established a systematic approach towards generation of metal oxide/metal sulfide/metal phosphide/metal nanoparticle, etc. embedded, highly conducting graphitic carbon material. Although, most of these materials lose the characteristic structure of the parent MOFs due to destruction of the framework by thermal treatment, but it is a notable approach to develop MOF derived OER electrocatalysts. One such OER electrocatalyst synthesized by annealing of a Co based MOF under air, was reported by Gong et al., where the active unit was $\mathrm{Co}_{3} \mathrm{O}_{4} \cdot{ }^{136}$ Apart from this, carbonization (under $\left.\mathrm{N}_{2}, \mathrm{H}_{2}, \mathrm{He}\right){ }^{137}$ thermal phosphidization, thermal sulphization, etc., can also result in further functional tuning. Employment of these techniques can help in the construction of various interesting structures, like functionalized carbon nano tubes, ${ }^{138}$ metal oxides embedded amorphous carbon matrix, porous nano-cages, ${ }^{139}$ double shell nano cages, ${ }^{140}$ etc., which can act as OER electrocatalysts. Combination of chemical etching, metal ion doping, hetero-atom doping and guest incorporation along with thermal treatment can result into enhanced functionality and stability. Most of these materials are reported to have high efficiency for OER electrocatalysis in basic $\mathrm{pH}$. However, in the true sense, these thermally derived materials using MOF as a precursor cannot be termed as MOFs, and thus, are beyond the scope of this article. In recent years, a handful of comprehensive and informative reviews have discussed and summarized the recent progress of thermally derived $\mathrm{C}-\mathrm{N}$ materials from MOFs and their application in the field of electrocatalysis. ${ }^{18,27,47,49,68,141-143}$

\section{Future scope of MOFs for OER electrocatalysis}

Some of the early reports on MOF-based OER electrocatalysts were associated with a few apparent shortcomings concerning the efficiency and/or stability of the catalysts. The design and fabrication of multi-metallic MOFs were found to be the notable strategic developments to evade these drawbacks and metalloligand based catalysis was one of the systematic and easiest ways to achieve that. In such systems, the metal center of the metalloligands can act as the catalytic center, while the metal node provides mechanical support. Incorporation of multiple metal ions in a MOF can also positively affect the OER catalytic efficiency by increasing the electrochemically active surface area (ECSA). This helps the system to achieve efficiency by increasing the number of coordinatively unsaturated metal sites in the framework, which then becomes accessible for catalysis. The performance of a MOF-based thin film as a catalyst largely depends upon the rate of mass transport and the electronic charge conduction. Unless a MOF-based catalyst possesses satisfactory charge transport, only a few layers of a MOF, adjacent to the electrode will be electrochemically active and can behave as a catalyst. On the other hand, to and fro diffusion of the substrate and products is essential in order to operate all the catalytically active sites of a MOF for any catalysis. Redox active metal node-containing 
thin layer MOFs can perform charge transport more efficiently than similar redox inactive MOFs, by a charge hopping mechanism. ${ }^{144}$ Similarly, MOFs having soft-soft interaction between the metal nodes and organic linkers, supramolecular $\pi-\pi$ interaction, composites with host-guest interaction ${ }^{145}$ and MOFs with high pore size ${ }^{59}$ can be a good carrier of charge. However, the combination of these desired features with the other essential requirements e.g., high stability under operational conditions and high activity of the catalytic species, requires reticular design strategies.

Strategies, like introducing a guest into a pristine framework to yield guest@MOF, have been developed to fabricate MOFbased catalytic systems. Depending upon the nature of the guest species, it can increase the charge transport ${ }^{146}$ of a catalytically active MOF framework. On the other hand, a catalytically active guest material can be incorporated inside a robust catalytically inactive MOF matrix to enhance the stability of the guest species and perform catalysis. These host-guest materials provide high scope for the modification of the electrocatalytic activity of the material by fine tuning the microenvironment around the guest species. Based on the recent works of our research group, it can be said that exploring the interfacial chemistry of different materials/molecules-MOF host-guest systems can result in better understanding of the structure-function relationship of the composite. Given its other merits, along with its ability to combine the efficiency of molecular catalysts and the robustness and stability of MOFs, a detailed investigation of such guest@MOF species would be interesting and might be of immense importance for the future design of effective electrocatalysts for clean energy applications. On the other hand, there are other candidates like MOF-based metal nanoparticle composites and core-shell structures, where the activity of the catalytic center is vastly influenced by the surrounding MOF which serves the dual benefit of stabilizing the catalyst as well as providing a porous support.

Furthermore, in order to tackle the issue of low electronic conductivity of most of the MOF-based OER electrocatalysts, there has been a rise in the use of conducting carbons (CNT, rGO, etc.), FTO, metal foams (Ni-, $\mathrm{Cu}-$, etc.) and layered double hydroxides $(\mathrm{LDH})$ as a sacrificial template, to grow mono- and/ or multi-metallic MOF nanoparticles. Various composites employing different guest species embedded into MOF thin films were reported to have one of the lowest overpotentials. Bearing the above merits in mind, designing and executing MOF-based composite material fabrication with other conducting and/or active catalytic species would be an effective strategy.

Thermally derived materials from MOFs presented an excellent scope to lower the inherent resistance and the overpotential requirement for the OER. The scope of reductive carbonization, oxidative calcination, phosphidation at an elevated temperature and many more reactions of graphitic carbons can lead to the generation of materials with diverse functionalities and with enhanced physical and chemical properties. This approach provides a huge scope of exploring various MOFs and MOF derivatives to prepare efficient and robust electrocatalysts for the OER.
This discussion clearly depicts the fact that various case specific modifications of MOFs to derive OER catalysts have led to a situation where generalizations can be formulated based on the structure-function relationship of MOFs and MOFderivatives. Careful observation suggests that a combination of the above-mentioned strategies can be highly effective to prepare better catalysts. For example, growing a thin film of guest@MOF composite on an electrode surface can result in a high TOF and low overpotential requirement for electrochemical OER. In various reports (a) metal ion-based engineering, (b) MNP@MOF, (c) MOF grown on electrode, etc. have been observed to be useful to prepare OER catalysts in combination with thermal treatment. To put it simply, each component of the MOF-derived composite has its own significant task and they all interact synergistically to enhance the overall electrocatalytic activity.

\section{Conclusion}

Thus, to conclude, it can be said that the MOFs hold high potential towards application in the field of electrochemical OER mainly because of their large surface area, pore volume, complex structure, and wide scope of functional tuning by means of structural modifications. Because of the huge range of choice of metal nodes, organic linkers and guest species, MOFs and MOF-derived materials hold a plethora of possibilities, very few of which have been explored. Moreover, since most of the MOFs are crystalline, it is easier to study the structure function relationships of the electrocatalysts. Looking back at the advancements made in this field in the last few years, we believe that the future of MOF-based electrocatalyst is beyond the realm of just MOFs. It requires a combination of various fields and research at the interface of different materials to ultimately prepare hybrid composites which can exploit the advantages of MOFs and their derivatives, leaving behind their limitations.

\section{Conflicts of interest}

There are no conflicts to declare.

\section{Acknowledgements}

We thank SERB, DST, Government of India (Project No. EMR/ 2017/002971) for the financial support. S. Mukhopadhyay thanks DST-INSPIRE, New Delhi, India and O. Basu thanks UGC, New Delhi, India for their fellowships. R. Nasani thanks UGC for Dr D S Kothari Postdoctoral fellowship (F.4-2/2006 (BSR)/CH/18-19/0032). We also thank DST-FIST, UGC-SAP, and UPE-II for the facilities. This work is also supported by UGCBSR-Mid-Career Award project (project No. 19-232/ 2019(BSR)).

\section{References}

1 P. E. Brockway, A. Owen, L. I. Brand-Correa and L. Hardt, Nat. Energy, 2019, 4, 612-621. 
2 D. Millstein, R. Wiser, M. Bolinger and G. Barbose, Nat. Energy, 2017, 2, 1-10.

3 B. M. Hunter, H. B. Gray and A. M. Müller, Chem. Rev., 2016, 116, 14120-14136.

4 W. Ruttinger and G. C. Dismukes, Chem. Rev., 1997, 97, 1-24.

5 C. Gao, J. Wang, H. Xu and Y. Xiong, Chem. Soc. Rev., 2017, 46, 2799-2823.

6 T. J. Meyer, M. V. Sheridan and B. D. Sherman, Chem. Soc. Rev., 2017, 46, 6148-6169.

7 J. Li, R. Güttinger, R. Moré, F. Song, W. Wan and G. R. Patzke, Chem. Soc. Rev., 2017, 46, 6124-6147.

8 Y. Xu, Q. Li, H. Xue and H. Pang, Coord. Chem. Rev., 2018, 376, 292-318.

9 J. G. McAlpin, T. A. Stich, W. H. Casey and R. D. Britt, Coord. Chem. Rev., 2012, 256, 2445-2452.

10 F. Kuttassery, S. Mathew, S. N. Remello, A. Thomas, K. Sano, Y. Ohsaki, Y. Nabetani, H. Tachibana and H. Inoue, Coord. Chem. Rev., 2018, 377, 64-72.

11 B. Singh and A. Indra, Chem. - Asian J., 2020, 607-623.

12 C. Freire, D. M. Fernandes, M. Nunes and V. K. Abdelkader, ChemCatChem, 2018, 10, 1703-1730.

13 B. D. McCarthy, A. M. Beiler, B. A. Johnson, T. Liseev, A. T. Castner and S. Ott, Coord. Chem. Rev., 2020, 406, 213137.

14 Q. Wang and D. Astruc, Chem. Rev., 2020, 120, 1438-1511.

15 A. Bavykina, N. Kolobov, I. S. Khan, J. A. Bau, A. Ramirez and J. Gascon, Chem. Rev., 2020, 120, 8468-8535.

16 J. Liu, S. Hou, W. Li, A. S. Bandarenka and R. A. Fischer, Chem. Asian J., 2019, 14, 3474-3501.

17 Q. Jiang, C. Zhou, H. Meng, Y. Han, X. Shi, C. Zhan and R. Zhang, J. Mater. Chem. A, 2020, 8, 15271-15301.

18 H. F. Wang, L. Chen, H. Pang, S. Kaskel and Q. Xu, Chem. Soc. Rev., 2020, 49, 1414-1448.

19 A. Dhakshinamoorthy, M. Alvaro and H. Garcia, Chem. Commun., 2012, 48, 11275-11288.

20 L. Kong, M. Zhong, W. Shuang, Y. Xu and X.-H. Bu, Chem. Soc. Rev., 2020, 49, 2378-2407.

21 S. Jin, ACS Energy Lett., 2019, 4, 1443-1445.

22 J. D. Blakemore, R. H. Crabtree and G. W. Brudvig, Chem. Rev., 2015, 115, 12974-13005.

23 M. Zhao, S. Ou and C. De Wu, Acc. Chem. Res., 2014, 47, 1199-1207.

24 P. Q. Liao, J. Q. Shen and J. P. Zhang, Coord. Chem. Rev., 2018, 373, 22-48.

25 Q. Shao, J. Yang and X. Huang, Chem. - Eur. J., 2018, 24, 15143-15155.

26 X. Hu and S. Dong, J. Mater. Chem., 2008, 18, 1279-1295.

27 Y. Z. Chen, R. Zhang, L. Jiao and H. L. Jiang, Coord. Chem. Rev., 2018, 362, 1-23.

28 P. Garrido-Barros, C. Gimbert-Suriñach, R. Matheu, X. Sala and A. Llobet, Chem. Soc. Rev., 2017, 46, 6088-6098.

29 Q. Ren, H. Wang, X. F. Lu, Y. X. Tong and G. R. Li, Adv. Sci., 2018, 5, 1700515.

30 C. A. Downes and S. C. Marinescu, ChemSusChem, 2017, 10, 4374-4392.

31 M. Shamsipur and A. Pashabadi, Coord. Chem. Rev., 2018, 374, 153-172.

32 H. Zhu, D. Liu, D. Zou and J. Zhang, J. Mater. Chem. A, 2018, 6, 6130-6154.

$33 \mathrm{~W}$. Li, S. Watzele, H. A. El-Sayed, Y. Liang, G. Kieslich, A. S. Bandarenka, K. Rodewald, B. Rieger and R. A. Fischer, J. Am. Chem. Soc., 2019, 141, 5926-5933.

34 S. Zhao, Y. Wang, J. Dong, C. T. He, H. Yin, P. An, K. Zhao, X. Zhang, C. Gao, L. Zhang, J. Lv, J. Wang, J. Zhang, A. M. Khattak, N. A. Khan, Z. Wei, J. Zhang, S. Liu, H. Zhao and Z. Tang, Nat. Energy, 2016, 1, 1-10.

35 M. B. Solomon, T. L. Church and D. M. D'Alessandro, CrystEngComm, 2017, 19, 4049-4065.

36 A. H. Chughtai, N. Ahmad, H. A. Younus, A. Laypkov and F. Verpoort, Chem. Soc. Rev., 2015, 44, 6804-6849.

37 H. Lv, Y. V. Geletii, C. Zhao, J. W. Vickers, G. Zhu, Z. Luo, J. Song, T. Lian, D. G. Musaev and C. L. Hill, Chem. Soc. Rev., 2012, 41, 7572-7589.

38 W. Cheng, X. Zhao, H. Su, F. Tang, W. Che, H. Zhang and Q. Liu, Nat. Energy, 2019, 4, 115-122.

39 C. Singh, I. Liberman, R. Shimoni, R. Ifraemov and I. Hod, J. Phys. Chem. Lett., 2019, 10, 3630-3636.
40 D. Gao, I. Trentin, L. Schwiedrzik, L. González and C. Streb, Molecules, 2020, 25, 1-20.

41 X. Zhou, T. Zhang, C. W. Abney, Z. Li and W. Lin, ACS Appl. Mater. Interfaces, 2014, 6, 18475-18479.

42 K. Chen and C. Wu, Coord. Chem. Rev., 2019, 378, 445-465.

43 Q. Y. Li, L. Zhang, Y. X. Xu, Q. Li, H. Xue and H. Pang, ACS Sustainable Chem. Eng., 2019, 7, 5027-5033.

44 B. A. Johnson, A. Bhunia and S. Ott, Dalton Trans., 2017, 46, 1382-1388.

45 S. Lin, P. M. Usov and A. J. Morris, Chem. Commun., 2018, 54, 6965-6974.

46 S. Wang, Y. Hou, S. Lin and X. Wang, Nanoscale, 2014, 6, 9930-9934.

47 W. Xia, A. Mahmood, R. Zou and Q. Xu, Energy Environ. Sci., 2015, 8, 1837-1866.

48 J. Juan-Alcañiz, J. Gascon and F. Kapteijn, J. Mater. Chem., 2012, 22, 10102-10119.

49 A. Mahmood, W. Guo, H. Tabassum and R. Zou, Adv. Energy Mater., 2016, 6, 1-26.

50 A. Dhakshinamoorthy and H. Garcia, Chem. Soc. Rev., 2012, 41, 5262-5284.

51 J. Liu and C. Wöll, Chem. Soc. Rev., 2017, 46, 5730-5770.

52 M. Batool, S. Ibrahim, B. Iqbal, S. Ali, A. Badshah, S. Abbas, D. R. Turner and M. A. Nadeem, Electrochim. Acta, 2019, 298, 248-253.

53 X.-F. Lu, L.-F. Gu, J.-W. Wang, J.-X. Wu, P.-Q. Liao and G.-R. Li, Adv. Mater., 2017, 29, 1604437.

54 H. B. Aiyappa, J. Masa, C. Andronescu, M. Muhler, R. A. Fischer and W. Schuhmann, Small Methods, 2019, 3, 1800415.

55 A. M. Ullman, J. W. Brown, M. E. Foster, F. Léonard, K. Leong, V. Stavila and M. D. Allendorf, Inorg. Chem., 2016, 55, 7233-7249.

56 Y. Xue, S. Zheng, H. Xue and H. Pang, J. Mater. Chem. A, 2019, 7, 7301-7327.

57 R. Zhu, J. Ding, Y. Xu, J. Yang, Q. Xu and H. Pang, Small, 2018, 14, 1803576.

58 B. A. Johnson, A. M. Beiler, B. D. McCarthy and S. Ott, J. Am. Chem. Soc., 2020, 142, 11941-11956.

59 M. Cai, Q. Loague and A. J. Morris, J. Phys. Chem. Lett., 2020, 11, 702-709.

60 S. Bose, J. Debgupta, R. M. Ramsundar and S. K. Das, Chem. - Eur. J., 2017, 23, 8051-8057.

61 C. Singh, S. Mukhopadhyay and S. K. Das, Inorg. Chem., 2018, 57, 6479-6490.

62 A. P. Wight and M. E. Davis, Chem. Rev., 2002, 102, 3589-3614.

63 C. Wang, Z. Xie, K. E. Dekrafft and W. Lin, J. Am. Chem. Soc., 2011, 133, 13445-13454.

64 O. Shekhah, J. Liu, R. A. Fischer and C. Wöll, Chem. Soc. Rev., 2011, 40, 1081-1106.

65 J. D. Evans, C. J. Sumby and C. J. Doonan, Chem. Soc. Rev., 2014, 43, 5933-5951.

66 Q. L. Zhu and Q. Xu, Chem. Soc. Rev., 2014, 43, 5468-5512.

67 K. Meyer, M. Ranocchiari and J. A. Van Bokhoven, Energy Environ. Sci., 2015, 8, 1923-1937.

68 N. Cheng, L. Ren, X. Xu, Y. Du and S. X. Dou, Adv. Energy Mater., 2018, 8, 1-21.

69 Y. Yan, T. He, B. Zhao, K. Qi, H. Liu and B. Y. Xia, J. Mater. Chem. A, 2018, 6, 15905-15926.

70 C. Guan, X. Liu, W. Ren, X. Li, C. Cheng and J. Wang, Adv. Energy Mater., 2017, 7, 1-8.

71 X. Li, S. Zheng, L. Jin, Y. Li, P. Geng, H. Xue, H. Pang and Q. Xu, Adv. Energy Mater., 2018, 8, 1-25.

72 H. R. Moon, D. W. Lim and M. P. Suh, Chem. Soc. Rev., 2013, 42, 1807-1824.

73 Q. Yang, Q. Xu and H. L. Jiang, Chem. Soc. Rev., 2017, 46, $4774-4808$.

74 S. K. Bhardwaj, N. Bhardwaj, R. Kaur, J. Mehta, A. L. Sharma, K. H. Kim and A. Deep, J. Mater. Chem. A, 2018, 6, 14992-15009.

75 O. Basu, S. Mukhopadhyay and S. K. Das, J. Chem. Sci., 2018, 130, $1-15$.

76 X. F. Lu, P. Q. Liao, J. W. Wang, J. X. Wu, X. W. Chen, C. T. He, J. P. Zhang, G. R. Li and X. M. Chen, J. Am. Chem. Soc., 2016, 138, 8336-8339.

77 Y. Gong, H. F. Shi, P. G. Jiang, W. Hua and J. H. Lin, Cryst. Growth Des., 2014, 14, 649-657. 
78 S. Gutiérrez-Tarriño, J. L. Olloqui-Sariego, J. J. Calvente, M. Palomino, G. M. Espallargas, J. L. Jorda, F. Rey and P. OñaBurgos, ACS Appl. Mater. Interfaces, 2109, 11, 46658-46665.

79 S. Ibrahim, K. Shehzadi, B. Iqbal, S. Abbas, D. R. Turner and M. A. Nadeem, J. Colloid Interface Sci., 2019, 545, 269-275.

80 J. Jiang, L. Huang, X. Liu and L. Ai, ACS Appl. Mater. Interfaces, 2017, 9, 7193-7201.

81 X. Wang, B. Li, Y.-P. Wu, A. Tsamis, H.-G. Yu, S. Liu, J. Zhao, Y.-S. Li and D.-S. Li, Inorg. Chem., 2020, 59, 4764-4771.

82 H. Dong, X. Zhang, X.-C. Yan, Y.-X. Wang, X. Sun, G. Zhang, Y. Feng and F.-M. Zhang, ACS Appl. Mater. Interfaces, 2019, 11, 45080-45086.

83 P. M. Usov, S. R. Ahrenholtz, W. A. Maza, B. Stratakes, C. C. Epley, M. C. Kessinger, J. Zhu and A. J. Morris, J. Mater. Chem. A, 2016, 4, 16818-16823.

84 K. Maity, K. Bhunia, D. Pradhan and K. Biradha, ACS Appl. Mater. Interfaces, 2017, 9, 37548-37553.

85 J. Gao, J. Cong, Y. Wu, L. Sun, J. Yao and B. Chen, ACS Appl. Energy Mater., 2018, 1, 5140-5144.

86 X. Zhao, B. Pattengale, D. Fan, Z. Zou, Y. Zhao, J. Du, J. Huang and C. Xu, ACS Energy Lett., 2018, 3, 2520-2526.

87 Y. Duan, S. Sun, S. Xi, X. Ren, Y. Zhou, G. Zhang, H. Yang, Y. Du and Z. J. Xu, Chem. Mater., 2017, 29, 10534-10541.

88 B. S. Yeo and A. T. Bell, J. Am. Chem. Soc., 2011, 133, 5587-5593.

89 F. Zheng, D. Xiang, P. Li, Z. Zhang, C. Du, Z. Zhuang, X. Li and W. Chen, ACS Sustainable Chem. Eng., 2019, 7, 9743-9749.

90 D. Zhang, R. Huang, H. Xie, R. Li, X. Liu, M. Pan and Y. Lei, Chem. Pap., 2020, 74, 2775-2784.

91 Z. Wan, D. Yang, J. Chen, J. Tian, T. T. Isimjan and X. Yang, ACS Appl. Nano Mater., 2019, 2, 6334-6342.

92 W. Li, W. Fang, C. Wu, K. N. Dinh, H. Ren, L. Zhao, C. Liu and Q. Yan, J. Mater. Chem. A, 2020, 8, 3658-3666.

93 J. Xu, Z. Xiao and X. Jia, ACS Sustainable Chem. Eng., 2019, 7, 6629-16639.

94 X. J. Bai, Y. N. Li, X. M. Yang, M. Y. Zhang, L. Shao, B. Zhang, T. Q. Wang, X. M. Zhang, L. Y. Zhang, Y. Fu and W. Qi, Chem. Commun., 2019, 55, 9343-9346.

95 Q. Qian, Y. Li, Y. Liu, L. Yu and G. Zhang, Adv. Mater., 2019, 31, 1-8.

96 Y. Gong, H. F. Shi, Z. Hao, J. L. Sun and J. H. Lin, Dalton Trans., 2013, 42, 12252-12259.

97 Y. Gong, Z. Hao, J. Meng, H. Shi, P. Jiang, M. Zhang and J. Lin, ChemPlusChem, 2014, 79, 266-277.

98 A. Bhunia, B. A. Johnson, J. Czapla-Masztafiak, J. Sá and S. Ott, Chem. Commun., 2018, 54, 7770-7773.

99 Z. Xue, Y. Li, Y. Zhang, W. Geng, B. Jia, J. Tang, S. Bao, H. P. Wang, Y. Fan, Z. wen Wei, Z. Zhang, Z. Ke, G. Li and C. Y. Su, Adv. Energy Mater., 2018, 8, 1801564.

100 D. Han, K. Huang, X. Li, M. Peng, L. Jing, B. Yu, Z. Chen and D. Qin, RSC Adv., 2019, 9, 33890-33897.

101 W. He, H.-M. Gao, R. Shimoni, Z.-Y. Lu and I. Hod, ACS Appl. Energy Mater., 2019, 2, 2138-2148.

102 A. Bucci, S. S. Mondal, V. Martin-Diaconescu, A. Shafir and J. Lloret-Fillol, ACS Appl. Energy Mater., 2019, 2, 8930-8938.

103 T. Ishida, M. Nagaoka, T. Akita and M. Haruta, Chem. - Eur. J., 2008, 14, 8456-8460.

104 T. Ishida, N. Kawakita, T. Akita and M. Haruta, Gold Bull., 2009, 42, 267-274.

105 S. B. Kalidindi, D. Esken and R. A. Fischer, Chem. - Eur. J., 2011, 17, 6594-6597.

106 J. Guo, X. Zhang, Y. Sun, L. Tang, Q. Liu and X. Zhang, ACS Sustainable Chem. Eng., 2017, 5, 11577-11583.

107 B. Nepal and S. Das, Angew. Chem., Int. Ed., 2013, 52, 7224-7227.

108 P. Manna, J. Debgupta, S. Bose and S. K. Das, Angew. Chem., Int. $E d ., 2016,55,2425-2430$.

109 S. Mukhopadhyay, J. Debgupta, C. Singh, A. Kar and S. K. Das, Angew. Chem., Int. Ed., 2018, 57, 1918-1923.

110 V. K. Abdelkader-Fernández, Diana M. Fernandes, S. S. Balula, L. Cunha-Silva and C. Freire, ACS Appl. Energy Mater., 2020, 3, 2925-2934.

111 S. Mukhopadhyay, O. Basu, A. Kar and S. K. Das, Inorg. Chem., 2020, 59, 472-483.
112 S. Mukhopadhyay, O. Basu and S. K. Das, ChemCatChem, DOI: 10.1002/cctc. 202000804 .

113 B. V. K. J. Schmidt, Macromol. Rapid Commun., 2020, 41, 1900333.

114 K. E. Ramohlola, G. R. Monana, M. J. Hato, K. D. Modibane, K. M. Molapo, M. Masikini, S. B. Mduli and E. I. Iwuoha, Composites, Part B, 2018, 137, 129-139.

115 K. E. Ramohlola, M. Masikini, S. B. Mdluli, G. R. Monama, M. J. Hato, K. M. Molapo, E. I. Iwuoha and K. D. Modibane, Int. J. Electrochem. Sci., 2017, 12, 4392-4405.

116 Y. Zhang, X. Feng, S. Yuan, J. Zhou and B. Wang, Inorg. Chem. Front., 2016, 3, 896-909.

117 W. J. Dang, Y. Q. Shen, M. Lin, H. Jiao, L. Xu and Z. L. Wang, J. Alloys Compd., 2019, 792, 69-76.

118 S. Sohrabi, S. Dehghanpour and M. Ghalkhani, J. Mater. Sci., 2018, 53, 3624-3639.

119 A. Xie, J. Du, F. Tao, Y. Tao, Z. Xiong, S. Luo, X. Li and C. Yao, Electrochim. Acta, 2019, 305, 338-348.

120 M. Jahan, Z. Liu and K. P. Loh, Adv. Funct. Mater., 2013, 23, 5363-5372.

121 Y. Fang, X. Li, F. Li, X. Lin, M. Tian, X. Long, X. An, Y. Fu, J. Jin and J. Ma, J. Power Sources, 2016, 326, 50-59.

122 L. Zhao, B. Dong, S. Li, L. Zhou, L. Lai, Z. Wang, S. Zhao, M. Han, K. Gao, M. Lu, X. Xie, B. Chen, Z. Liu, X. Wang, H. Zhang, H. Li, J. Liu, H. Zhang, X. Huang and W. Huang, ACS Nano, 2017, 11, 5800-5807.

123 Y. Wen, Z. Wei, C. Ma, X. Xing, Z. Li and D. Luo, Nanomaterials, 2019, 9, 775.

124 X. Deng and H. Tüysüz, ACS Catal., 2014, 4, 3701-3714.

125 B. Chakraborty and I. A. Weinstock, Coord. Chem. Rev., 2019, 382, 85-102.

126 J. Wang and H. C. Zeng, ACS Appl. Mater. Interfaces, 2019, 11, 23180-23191.

127 Z. Gao, Z. W. Yu, F. Q. Liu, Y. Yu, X. M. Su, L. Wang, Z. Z. Xu, Y. L. Yang, G. R. Wu, X. F. Feng and F. Luo, Inorg. Chem., 2019, 58, 11500-11507.

128 W. Zhang, Y. Wang, H. Zheng, R. Li, Y. Tang, B. Li, C. Zhu, L. You, M.-R. Gao, Z. Liu, S.-H. Yu and K. Zhou, ACS Nano, 2020, 14, 1971-1981.

129 K. Srinivas, Y. Lu, Y. Chen, W. Zhang and D. Yang, ACS Sustainable Chem. Eng., 2020, 8, 3820-3831.

130 S. Pintado, S. Goberna-Ferrón, E. C. Escudero-Adán and J. R. GalánMascarós, J. Am. Chem. Soc., 2013, 135, 13270-13273.

131 W. Ye, Y. Yang, X. Fang, M. Arif, X. Chen and D. Yan, ACS Sustainable Chem. Eng., 2019, 7, 18085-18092.

132 X. Ling, F. Du, Y. Zhang, Y. Shen, T. Li, A. Alsaedi, T. Hayat, Y. Zhou and Z. Zou, RSC Adv., 2019, 9, 33558-33562.

133 J. Du, S. Xu, L. Sun and F. Li, Chem. Commun., 2019, 55, 14773-14776.

134 L. Huang, G. Gao, H. Zhang, J. Chen, Y. Fang and S. Dong, Nano Energy, 2020, 68, 104296.

135 B. Ye, R. Jiang, Z. Yu, Y. Hou, J. Huang, B. Zhang, Y. Huang, Y. Zhang and R. Zhang, J. Catal., 2019, 380, 307-317.

136 Y. Gong, Z. Xu and H. Pan, ChemistrySelect, 2019, 4, 1131-1137.

137 R. Xiang, Y. Duan, C. Tong, L. Peng, J. Wang, S. S. A. Shah, T. Najam, X. Huang and Z. Wei, Electrochim. Acta, 2019, 302, 45-55.

138 T. Y. Ma, S. Dai, M. Jaroniec and S. Z. Qiao, J. Am. Chem. Soc., 2014, 136, 13925-13931.

139 Y. Hou, J. Li, Z. Wen, S. Cui, C. Yuan and J. Chen, Nano Energy, 2015, 12, 1-8.

140 H. Hu, B. Guan, B. Xia and X. W. Lou, J. Am. Chem. Soc., 2015, 137, 5590-5595.

141 Q. Shi, S. Fu, C. Zhu, J. Song, D. Du and Y. Lin, Mater. Horiz., 2019, 6, 684-702.

142 J. K. Sun and Q. Xu, Energy Environ. Sci., 2014, 7, 2071-2100.

143 J. Zhang, H. Li, P. Guo, H. Ma and X. S. Zhao, J. Mater. Chem. A, 2016, 4, 8497-8511.

144 L. Sun, C. H. Hendon, S. S. Park, Y. Tulchinsky, R. Wan, F. Wang, A. Walsh and M. Dincă, Chem. Sci., 2017, 8, 4450-4457.

145 S. Lin, P. M. Usov and A. J. Morris, Chem. Commun., 2018, 54, 6965-6974.

146 Y. Kobayashi, B. Jacobs, M. D. Allendorf and J. R. Long, Chem. Mater., 2010, 22, 4120-4122. 